\title{
$\$$ Research Square \\ Bifurcation Analysis of Rotor/Bearing System using Third Order Journal Bearings Stiffness and Damping Coefficients
}

Tamer Elsayed ( $\boldsymbol{\nabla}$ tamer.el-sayed@abdn.ac.uk)

University of Aberdeen https://orcid.org/0000-0002-1494-5334

Hussein Sayed

Helwan University

\section{Research Article}

Keywords: Journal bearing, bearing coeffcient, infinitesimal perturbation, rotordynamics, Jeffcott rotor, numerical solution.

Posted Date: March 1st, 2021

DOI: https://doi.org/10.21203/rs.3.rs-227710/v1

License: (c) (i) This work is licensed under a Creative Commons Attribution 4.0 International License.

Read Full License 


\title{
Bifurcation Analysis of Rotor/Bearing System using Third Order Journal Bearings Stiffness and Damping Coefficients
}

\author{
T.A. El-Sayed ${ }^{\mathrm{a}, \mathrm{b}, *}$, Hussein Sayed $^{\mathrm{b}}$ \\ ${ }^{a}$ Centre for Applied Dynamics Research, School of Engineering, University of Aberdeen, Aberdeen, AB24 \\ उUE, $U K$ \\ ${ }^{b}$ Department of Mechanical Design, Faculty of Engineering, Mataria, Helwan University, P.O. Box 11718, \\ Helmeiat-Elzaton, Cairo, Egypt
}

\begin{abstract}
Journal bearings have many applications in industry due to its high load carrying capacity. In addition proper design of journal bearings enables safe operation at very high speeds. However, they are susceptible to oil whirl instability which may cause bearing failure. The fluid film pressure distribution inside the journal bearing is described by Reynolds equation. Many studies had been done to approximate the bearing performance using first order bearing coefficients. Although this analysis is stable for evaluating the threshold speed but it is insensitive to limit cycles above the threshold speed. Mush literature show that above the threshold speed, subcritical or supercritical bifurcations may be observed. Therefore, the aim of the present paper is to evaluate the third order bearing coefficients for a finite length journal bearing using finite perturbation method. The values of these coefficients are evaluated using infinitesimal perturbation analysis. These values are used to investigate the bifurcation stability of flexible Jeffcott rotor supported by two symmetric journal bearings. The effect of rotor stiffness ratio on the bifurcation stability of the system is investigated. The results of this work show that the third order parameters can be used to evaluate the type of bifurcation above the threshold speed.
\end{abstract}

Keywords: Journal bearing, bearing coefficient, infinitesimal perturbation, rotordynamics, Jeffcott rotor, numerical solution.

\footnotetext{
*Corresponding author

Email addresses: tamer.elsayed@abdn.ac.uk (T.A. El-Sayed), husseinsayed2008@m-eng.helwan.edu.eg (Hussein Sayed)
} 
c

$c_{i j}$

$c_{i j k}$

$c_{i j k l}$

$C_{i j}$

$C_{i j k}$

$C_{i j k l}$

e

$F_{x}, F_{y}$

$\bar{F}_{X}, \bar{F}_{Y}$

$h$

H

$k_{i j}$

$k_{i j k}$

$k_{i j k l}$

$K_{i j}$

$K_{i j k}$

$K_{i j k l}$

$m$

$m_{d}$

$m_{j}$

$\bar{M}$

$\bar{M}_{t h}$

$p$

$P$

$P_{0}$

$P_{\zeta}$

$P_{\zeta \eta}$

$P_{\zeta \eta \gamma}$

$R$

$S$

$T$

$X, Y$

$X^{\prime}, Y^{\prime}, X^{\prime \prime}, Y^{\prime \prime}$ $z$

$\varepsilon$

$\theta$

$\theta_{1}$

$\mu$

$\rho$

$\tau$

$\phi$

$\Omega$
Journal bearing radial clearance

First order damping coefficient (N.s/m), $i, j=x, y$

Second order damping coefficient $\left(\mathrm{N} . \mathrm{s} / \mathrm{m}^{2}\right), i, j, k, l=x, y$

Third order damping coefficient $\left(\mathrm{N} . \mathrm{s} / \mathrm{m}^{3}\right), i, j, k=x, y$

Dimensionless linear damping coefficients $C_{i j}=\frac{c_{i j} c \Omega}{W}$

Dimensionless second order damping coefficients $C_{i j k l}=\frac{c_{i j k} c^{2} \Omega}{W}$

Dimensionless third order damping coefficients $C_{i j k}=\frac{c_{i j k l} c^{b^{2}} \Omega}{W}$

Radial eccentericity

Bearing forces components in Cartesian coordinates $\mathrm{N}$

Dimensionless bearing forces components $\bar{F}_{X}=\frac{F_{x}}{W}, \bar{F}_{Y}=\frac{F_{y}}{W}$

Thickness of the fluid film (m)

Fluid film dimensionless thickness $H=\frac{h}{c}$

$1^{\text {st }}$ order stiffness coefficients $(\mathrm{N} / \mathrm{m}), i, j=x, y$

$2^{\text {nd }}$ order Stiffness coefficients $\left(\mathrm{N} / \mathrm{m}^{2}\right), i, j, k=x, y$

$3^{\text {rd }}$ order stiffness coefficients $\left(\mathrm{N} / \mathrm{m}^{3}\right), i, j, k, l=x, y$

$1^{\text {st }}$ order dimensionless stiffness coefficients $K_{i j}=\frac{k_{i j} c}{W}$

$2^{\text {nd }}$ order dimensionless stiffness coefficients $K_{i j k}=\frac{k c^{2}}{W_{0}}$

$3^{\text {rd }}$ order dimensionless stiffness coefficients $K_{i j k l}=\frac{k c^{3}}{W}$

Total mass of the disc and shaft

Sum of disc and shaft around journal mass

Mass of shaft around the journal

dimensionless mass $\bar{M}=\frac{m c \Omega^{2}}{W}$

Threshold mass $\bar{M}_{t h}=\frac{m \text { th } \Omega_{t h}^{2}}{{ }^{W}}$

Fluid film pressure $\left(\mathrm{N} / \mathrm{m}^{2}\right)$

Dimensionless pressure $P=\frac{p}{6 \mu \Omega}\left(\frac{c}{R}\right)^{2}$

Dimensionless steady state pressure

$1^{\text {st }}$ order pressure gradients $\zeta=x, y$

$2^{\text {nd }}$ order pressure gradients $\zeta, \eta=x, y$

$3^{\text {rd }}$ order pressure gradients $\zeta, \eta, \gamma=x, y$

Bearing Radius (m)

Sommerfeld number

periodic time

Dimensionless displacements

Cartesian dimensionless velocities and accelerations.

Axial coordinate

Eccentricity ratio $(e / c)$

attitude angle

small perturbation of attitude angle

lubricant viscosity (N.s/m $\left.{ }^{2}\right)$

Lubricant density $\left(\mathrm{kg} / \mathrm{m}^{3}\right)$

dimensionless time $(\tau=\Omega t)$

the angular coordinate

the rotational speed of the journal rad/s 


\section{Introduction}

Journal bearing is one of the crucial elements used in the engineering industry. It has many applications in heavy-duty ,moderate and high speed machinery such as reciprocating engines (Santos et al., Chun and Khonsari 2016), turbomachines (Salamone 1984, Ciulli et al. 2018, On et al. 2018), centrifugal pumps(Bai et al. 2019, Nishimura et al. 2018) and turbocharger (El-Sayed and Fatah 2016, Smolík et al. 2017, Novotný et al. 2018), etc. Many of these machines are required to spin at very high speeds to improve their efficiency. Therefore, it is important to investigate and evaluate the threshold whirling speed (Ehrich 1991. Vance et al. 2010). After this threshold oil whirl may be occurred. However, some applications are reported to operate at speeds higher than this threshold value in stable condition(Lund 1974). In addition, the experimental work of Muszynska (1988), Deepak and Noah (1998) showed that a stable whirling is occurred after threshold speed. Therefore, it is important to investigate the nonlinear behavior of journal bearings to improve the future design of rotating machinery.

The dynamic modelling of journal bearings requires the evaluation of the bearing forces. Since the fluid film pressure distribution inside the journal bearing can be described by the Reynolds equation, the bearing forces can be obtained from the integration of the pressure distribution inside the bearing. In case of short and long bearings, Reynolds equation can be simplified so that the bearing forces can be obtained analytically, see for example (Castro et al., Huang et al. 2017, Nishimura et al. 2018). Nishimura et al. (2018) investigated the nonlinear dynamics and stability of a vertical flexible rotor supported by short journal bearing. The bearing forces are evaluated and approximated based on the short bearing formula presented by Mori (1968). Castro et al. investigated the linear dynamics of rotors supported by short journal bearings. They investigated the instability 'oil whirl' and 'oil whip' during run-up and run-down. In case of finite bearing length where the analytical solution for Reynolds equation is not available, the bearing forces can be obtained by direct 
integration of Reynolds equation. In dynamic analysis, this integration is required to be done each time step which is computationally expensive especially if very fine mesh is considered Akers et al. Schweizer, Shin and Palazzolo 2020.

Alternatively, and more commonly is to represent the bearing using stiffness and damping coefficients. These coefficients are used to evaluate the bearing forces and to investigate the system dynamics. These coefficients are simply based on the Taylor expansion of the bearing forces. Much research considers only the linear first order approximations while others consider higher order approximation (Lund 1965, Lund and Thomsen 1978, Qiu and Tieu 1996, Miura et al. 2017). There are different methods to obtain the bearing coefficients,such as, experimental (Tiwari et al. 2004, Mao et al. 2016) analytical (Chasalevris and Sfyris, Wang and Khonsari 2006, Miraskari et al. 2018a), finite difference method(FDM) (Gero and McC. Ettles 1986, Qiu and Tieu 1995, Goodwin et al. 1997, Ebrat et al.), finite element method (FEM) (Wada et al. 1971, Mourelatos 1985, Arregui and Vázquez 2001, Nair and Nair 2004, Awasthi et al. 2006, Hili et al. 2010) , mesh less method with radial basis (MMRB) (Nicoletti 2013) and computational fluid dynamics (CFD) (Gao et al. 2014, Zhang et al. 2015, Gao et al. 2016, Zhang et al. 2016).

Investigating the Hopf bifurcation stability is important because it represents the transformation from spin motion to periodic whirl motion. Although the bearing force linear first order approximation coefficients are sufficient to obtain the threshold speed, but they are not sufficient to judge the stability of periodic solution and whether it is subcritical or super critical. This motivates the research is to either use analytical solution for bearing forces where possible or using higher order bearing coefficients.

Dakel et al. (2014) investigated the nonlinear dynamics and stability of a rotor mounted hydrodynamic journal bearing. They investigated the stability based on both linear bearing coefficients and on bearing force from the analytical solution. Smolík et al. (2019) investigated the stability of rotor bearing system based on the threshold stability curves. They 
used different approaches to calculate the bearing forces, infinitely short, infinitely short approximation with correction factor for finite length bearing, finite difference, and finite element. They also investigated the run-up time and acceleration.

Yu et al. (2005) introduced a time perturbation method to evaluate the bearing first order coefficient for aerodynamic compliant foil bearings. Weimin et al. (2012) extended a time perturbation method to evaluate the second order nonlinear coefficient for journal bearings. Miraskari et al. (2018b) investigated the nonlinear dynamics of flexible rotor supported by journal bearings. the second order nonlinear bearing coefficients are obtained using perturbation method. Then they used a shooting method to find the stability of the periodic solution. Chasalevris (2020) investigated the Hopf bifurcation of flexible rotor supported by journal bearings. He investigated the lemon bore and partial arc types of bearings. Chasalevris used Hopf bifurcation theory introduced by Hassard et al. (1981) to investigate the stability of periodic solution.

The previous literature shows that many studies for the nonlinear dynamics of journal bearing are based on the analytical solution which is only available in case of short or long bearings. Little studies are done based on the second order linear coefficient. Moreover, there is very little studies have done on higher order bearing coefficients. The main purpose of this study is to evaluate the third order bearing coefficient using finite perturbation method. The obtained coefficients are used to investigate the stability of a flexible rotor/disc supported on two symmetric hydrodynamic journal bearings.

After this introduction, the steps of how to obtain the journal bearing third order parameters are presented on the mathematical model section. Then the dynamic model of rotor supported by journal bearings is presented. The values of the obtained journal bearing coefficients are presented on the results and discussion section. The stability of the dynamical model is discussed at the dynamical results subsection. Finally, the sound results and conclusions are presented in conclusion section. 


\section{Analytical Model}

The present model is for full circular journal bearing $o_{b}$ is the center of the bearing, $o_{j s}$ is the center of the journal or shaft at the equilibrium position and $o_{j}$ is the perturbed journal center. $R$ is the radius of the journal bearing and $R_{j}$ is the radius of the journal. The radial clearance is $c=R-R_{j}$. The height of the oil film between the journal and the bearing at any angle is $h . e_{0}, \theta_{0}$ are the eccentricity and the attitude angle at the steady state equilibrium. $O_{j s}$ is the center of the journal or shaft at the equilibrium position in terms of dimensionless coordinates and $O_{j}$ is the perturbed journal center in terms of dimensionless coordinates , see Figure 1 $\mathrm{fc}$.

(a)

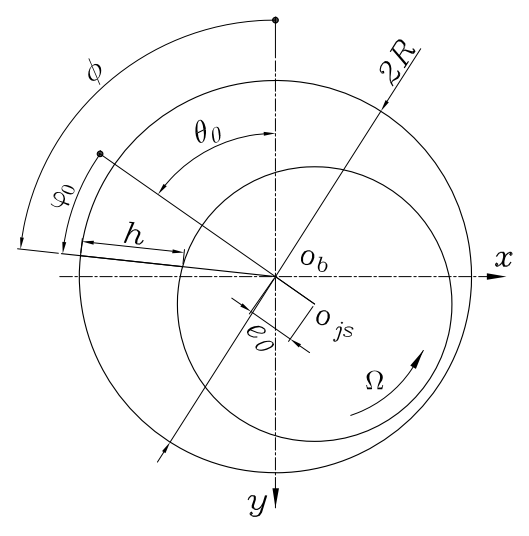

(b)

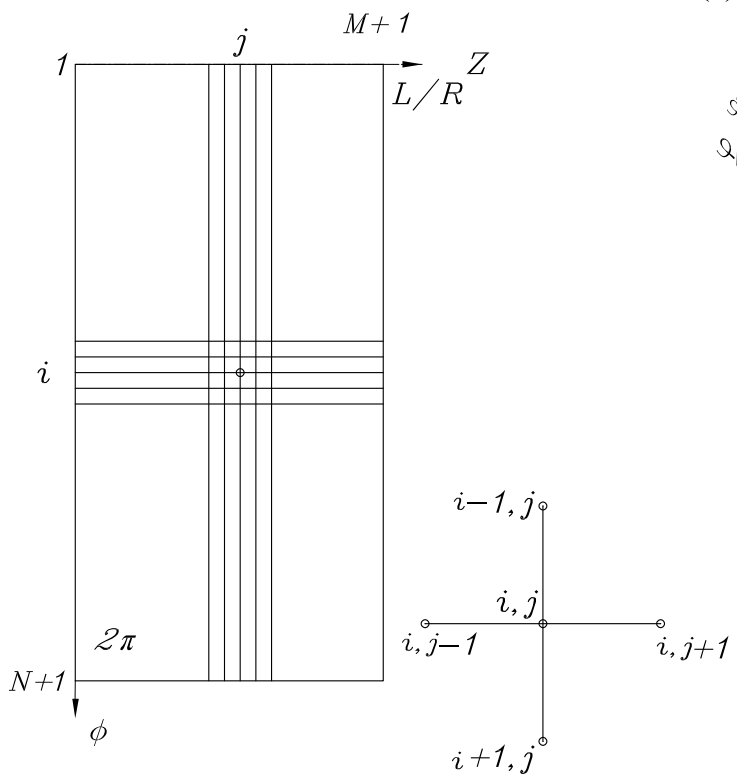

(c)

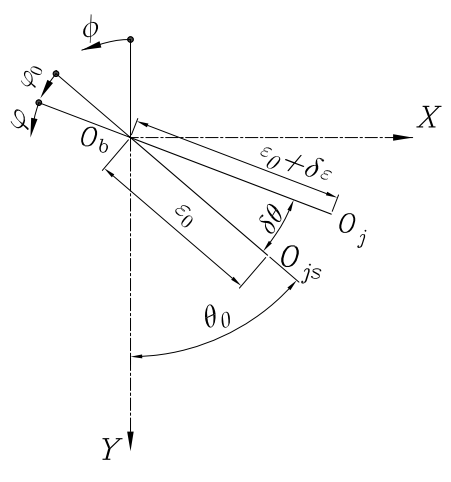

Figure 1: Full circular journal bearing (a) schematic of the journal bearing coordinates (b) journal bearing mesh (c) perturbed journal bearing in dimensionless coordinates $X$ and $Y$

The pressure distribution inside the fluid film can be described by Reynolds equation as follows:

$$
\frac{1}{R^{2}} \frac{\partial}{\partial \phi}\left(\frac{\rho h^{3}}{12 \mu} \frac{\partial p}{\partial \phi}\right)+\frac{\partial}{\partial z}\left(\frac{\rho h^{3}}{12 \mu} \frac{\partial p}{\partial z}\right)=\frac{\Omega}{2} \frac{\partial(\rho h)}{\partial \phi}+\rho \frac{\partial h}{\partial t}
$$


where $h$ and $p$ are the oil film thickness and pressure respectively. $\phi$ is the angular coordinate with respect to vertical axis, $t$ is the time in sec., $\Omega$ is the rotational speed of the shaft in $\mathrm{rad} / \mathrm{sec} . . x$ and $y$ are the sectional bearing coordinates as shown in Figure 1 -a. $\mathrm{z}$ is the axial coordinate as shown in Figure 1 -b. The fluid film viscosity and density are $\mu$ in N.s $/ \mathrm{m}^{2}$ and $\rho$ in $\mathrm{kg} / \mathrm{m}^{3}$ respectively.

Reformulating the Reynolds equation Eq. (1) for the dimensionless representation, it can be written as:

$$
\frac{\partial}{\partial \phi}\left(H^{3} \frac{\partial P}{\partial \phi}\right)+\frac{\partial}{\partial Z}\left(H^{3} \frac{\partial P}{\partial Z}\right)=\frac{\partial H}{\partial \phi}+2 \frac{\partial H}{\partial \tau}
$$

$H$ is the oil film dimensionless thickness, $P=\frac{p}{6 \mu \Omega}\left(\frac{c}{R}\right)^{2}$ is the dimensionless pressure, $Z=\frac{z}{R}$ is dimensionless location in axial direction. The dimensionless time is $\tau=\Omega \mathrm{t}$. To evaluate the bearing coefficients a small perturbation is introduced from the steady state point as shown in Figure 1 1 c. The height in dimensionless can be presented as in Eq.(3). After perturbation the eccentricity ratio, attitude angle and dimensionless height can be obtained from Eqs.(4), (5) and (6) respectively. Since the analysis in the present article is based on the third order approximation, All the differences with order higher than three is omitted.

$$
H=1+\varepsilon \cos (\phi-\theta)=1+\varepsilon \cos (\varphi)
$$

For which $\varepsilon$ is the eccentricity ratio and $\theta$ is the attitude angle, for a small perturbation

$$
\begin{aligned}
\varepsilon & =\varepsilon_{0}+\delta \varepsilon \\
\theta & =\theta_{0}+\delta \theta \\
H & =1+\left(\varepsilon_{0}+\delta \varepsilon\right) \cos \left(\varphi_{0}-\delta \theta\right)=1+\left(\varepsilon_{0}+\delta \varepsilon\right)\left[\cos \left(\varphi_{0}\right) \cos (\delta \theta)+\sin \left(\varphi_{0}\right) \sin (\delta \theta)\right]
\end{aligned}
$$


For small $\delta \theta$, substitute $\cos (\delta \theta)=1-\frac{\delta \theta^{2}}{2}$ and $\sin (\delta \theta)=\delta \theta-\frac{\delta \theta^{3}}{6}$, then equation (6) can be written as

$$
H=1+\left(\varepsilon_{0}+\delta \varepsilon\right)\left[\cos \left(\varphi_{0}\right)\left(1-\frac{\delta \theta^{2}}{2}\right)+\sin \left(\varphi_{0}\right)\left(\delta \theta-\frac{\delta \theta^{3}}{6}\right)\right]
$$

Equation (7) can be expanded as follows

$$
\begin{aligned}
H= & 1+\varepsilon_{0} \cos \left(\varphi_{0}\right)+\varepsilon_{0} \delta \theta \sin \left(\varphi_{0}\right)+\delta \varepsilon \cos \left(\varphi_{0}\right)+\delta \varepsilon \delta \theta \sin \left(\varphi_{0}\right)- \\
& \frac{1}{2} \varepsilon_{0} \delta \theta^{2} \cos \left(\varphi_{0}\right)-\frac{\delta \theta^{2}}{2} \delta \varepsilon \cos \left(\varphi_{0}\right)-\varepsilon_{0} \frac{\delta \theta^{3}}{6} \sin \left(\varphi_{0}\right)-\frac{\delta \varepsilon \delta \theta^{3}}{6} \sin \left(\varphi_{0}\right) \\
= & H_{0}+H_{1}+H_{2}+H_{3}+O\left(\delta \theta^{4}\right)
\end{aligned}
$$

for which

$$
\begin{aligned}
& H_{0}=1+\varepsilon_{0} \cos \left(\varphi_{0}\right) \\
& H_{1}=\varepsilon_{0} \delta \theta \sin \left(\varphi_{0}\right)+\delta \varepsilon \cos \left(\varphi_{0}\right) \\
& H_{2}=\delta \varepsilon \delta \theta \sin \left(\varphi_{0}\right)-\frac{1}{2} \varepsilon_{0} \delta \theta^{2} \cos \left(\varphi_{0}\right) \\
& H_{3}=-\frac{\delta \theta^{2}}{2} \delta \varepsilon \cos \left(\varphi_{0}\right)-\varepsilon_{0} \frac{\delta \theta^{3}}{6} \sin \left(\varphi_{0}\right)
\end{aligned}
$$

Now it is important to transform the height equation from polar coordinates to Cartesian coordinates. The transformation relations from $\varepsilon$ and $\theta$ coordinates to $X$ and $Y$ coordinates are as follows:

$$
\begin{aligned}
\delta \varepsilon & =\delta X \sin \left(\theta_{0}\right)+\delta Y \cos \left(\theta_{0}\right) \\
\varepsilon_{0} \delta \theta & =\delta X \cos \left(\theta_{0}\right)-\delta Y \sin \left(\theta_{0}\right)
\end{aligned}
$$


Substituting by the transformation relations in Eqs. (13) and (14) into Eqs. (9) to (12), results in

$$
\begin{aligned}
H_{0}= & 1+\varepsilon_{0} \cos \left(\varphi_{0}\right) \\
H_{1}= & \delta X \sin \phi+\delta Y \cos \phi \\
H_{2}= & \frac{\delta X^{2}}{2 \varepsilon_{0}}\left(2 \sin \theta_{0} \cos \theta_{0} \sin \varphi_{0}-\cos ^{2} \theta_{0} \cos \varphi_{0}\right) \\
& +\frac{\delta X \delta Y}{\varepsilon_{0}}\left(\sin \theta_{0} \cos \theta_{0} \cos \varphi_{0}+\cos ^{2} \theta_{0} \sin \varphi_{0}-\sin ^{2} \theta_{0} \sin \varphi_{0}\right) \\
& +\frac{\delta Y^{2}}{2 \varepsilon_{0}}\left(-2 \sin \theta_{0} \cos \theta_{0} \sin \varphi_{0}-\sin ^{2} \theta_{0} \cos \varphi_{0}\right)
\end{aligned}
$$

The dimensionless pressure distribution $P\left(X, Y, X^{\prime}, Y^{\prime}\right)$ in the fluid film can be expanded using Taylor expansion as follows:

$$
\begin{aligned}
P= & P_{0}+P_{X} \delta X+P_{Y} \delta Y+P_{X^{\prime}} \delta X^{\prime}+P_{Y^{\prime}} \delta Y^{\prime}+0.5 P_{X X} \delta X^{2}+0.5 P_{Y Y} \delta Y^{2} \\
& +P_{X Y} \delta X \delta Y+P_{X X^{\prime}} \delta X \delta X^{\prime}+P_{X Y^{\prime}} \delta X \delta Y^{\prime}+P_{Y X^{\prime}} \delta Y \delta X^{\prime}+P_{Y Y^{\prime}} \delta Y \delta Y^{\prime} \\
& +\frac{1}{6} P_{X X X} \delta X^{3}+\frac{1}{6} P_{Y Y Y} \delta Y^{3}+\frac{1}{2} P_{X X Y} \delta X^{2} \delta Y+\frac{1}{2} P_{X Y Y} \delta X \delta Y^{2} \\
& +\frac{1}{2} P_{X X X^{\prime}} \delta X^{2} \delta X^{\prime}+\frac{1}{2} P_{X X Y^{\prime}} \delta X^{2} \delta Y^{\prime}+\frac{1}{2} P_{Y Y X^{\prime}} \delta Y^{2} \delta X^{\prime} \\
& +\frac{1}{2} P_{Y Y Y^{\prime}} \delta Y^{2} \delta Y^{\prime}+P_{X Y X^{\prime}} \delta X \delta Y \delta X^{\prime}+P_{X Y Y^{\prime}} \delta X \delta Y \delta Y^{\prime}
\end{aligned}
$$

where,

$X=\frac{x}{c}, Y=\frac{y}{c},{ }^{\prime}=\frac{\mathrm{d}}{\mathrm{d} \tau}, \tau=\Omega t, X^{\prime}=\frac{x}{\Omega c}, Y^{\prime}=\frac{y}{\Omega c}$ $P_{X}=\frac{\partial P}{\partial X}, P_{X X}=\frac{\partial^{2} P}{\partial X^{2}}, P_{X X X}=\frac{\partial^{3} P}{\partial X^{3}}, P_{X Y Y^{\prime}}=\frac{\partial^{3} P}{\partial X \partial Y \partial Y^{\prime}}$ and so on.

To obtain the steady state pressure $P_{0}$ the Reynolds equation in Eq. (2) are solved using finite difference relations. Then to obtain the first order pressure gradient the Reynolds Eq. (2) is differentiated with respect to $X, Y, X^{\prime}$ and $Y^{\prime}$ and rearranged as shown in Eq. 19. Similarly, the second order pressure gradient can be obtained by differentiating 
the Reynolds first derivative equations in Eq. 19. with respect to $X, Y, X^{\prime}$ and $Y^{\prime}$ as shown in Eq.20 . Likewise, the third order pressure gradient in Eq. (18) can be obtained by differentiating the second order derivative of Eq. 20 with respect to $X, Y, X^{\prime}$ and $Y^{\prime}$ as shown in Eqs. 21) to (30). Solving Eqs. (19) to (30) using finite difference the first, second and third order pressure gradient can be obtained. It is worth noting that during the solution of these equations the first, second and third dimensionless height are required which are listed in Appendix A.

$$
\begin{array}{ll}
\frac{\partial}{\partial \phi}\left(H^{3} \frac{\partial P^{*}}{\partial \phi}\right)+\frac{\partial}{\partial Z}\left(H^{3} \frac{\partial P^{*}}{\partial Z}\right)= & P^{*}=P_{0} \\
\frac{\partial H_{0}}{\partial \phi} & P^{*}=P_{X} \\
\frac{\partial H_{X}}{\partial \phi}-\frac{\partial}{\partial \phi}\left(3 H_{0}^{2} H_{X} \frac{\partial P_{0}}{\partial \phi}\right)-\frac{\partial}{\partial Z}\left(3 H_{0}^{2} H_{X} \frac{\partial P_{0}}{\partial Z}\right) & P^{*}=P_{Y} \\
\frac{\partial H_{Y}}{\partial \phi}-\frac{\partial}{\partial \phi}\left(3 H_{0}^{2} H_{Y} \frac{\partial P_{0}}{\partial \phi}\right)-\frac{\partial}{\partial Z}\left(3 H_{0}^{2} H_{Y} \frac{\partial P_{0}}{\partial Z}\right) & P^{*}=P_{X^{\prime}} \\
2 H_{X} & P^{*}=P_{Y^{\prime}} \\
2 H_{Y} &
\end{array}
$$




$$
\begin{array}{ll}
\frac{\partial}{\partial \phi}\left(H^{3} \frac{\partial P^{*}}{\partial \phi}\right)+\frac{\partial}{\partial Z}\left(H^{3} \frac{\partial P^{*}}{\partial Z}\right)= & \\
\quad \frac{\partial H_{X X}}{\partial \phi}-\frac{\partial}{\partial \phi}\left[6 H_{0}^{2} H_{X} \frac{\partial P_{X}}{\partial \phi}+\left(6 H_{0} H_{X}^{2}+3 H_{0}^{2} H_{X X}\right) \frac{\partial P_{0}}{\partial \phi}\right] & P^{*}=P_{X X} \\
\quad-\frac{\partial}{\partial Z}\left[6 H_{0}^{2} H_{X} \frac{\partial P_{X}}{\partial Z}+\left(6 H_{0} H_{X}^{2}+3 H_{0}^{2} H_{X X}\right) \frac{\partial P_{0}}{\partial Z}\right] & \\
\quad \frac{\partial H_{Y Y}}{\partial \phi}-\frac{\partial}{\partial \phi}\left[6 H_{0}^{2} H_{Y} \frac{\partial P_{Y}}{\partial \phi}+\left(6 H_{0} H_{Y}^{2}+3 H_{0}^{2} H_{Y Y}\right) \frac{\partial P_{0}}{\partial \phi}\right] & P^{*}=P_{Y Y} \\
-\frac{\partial}{\partial Z}\left[6 H_{0}^{2} H_{Y} \frac{\partial P_{Y}}{\partial Z}+\left(6 H_{0} H_{Y}^{2}+3 H_{0}^{2} H_{Y Y}\right) \frac{\partial P_{0}}{\partial Z}\right] & \\
\frac{\partial H_{X Y}}{\partial \phi}-\frac{\partial}{\partial \phi}\left[3 H_{0}^{2} H_{Y} \frac{\partial P_{X}}{\partial \phi}+3 H_{0}^{2} H_{X} \frac{\partial P_{Y}}{\partial \phi}+\left(6 H_{0} H_{X} H_{Y}+3 H_{0}^{2} H_{X Y}\right) \frac{\partial P_{0}}{\partial \phi}\right] & P^{*}=P_{X Y} \\
-\frac{\partial}{\partial Z}\left[3 H_{0}^{2} H_{Y} \frac{\partial P_{X}}{\partial Z}+3 H_{0}^{2} H_{X} \frac{\partial P_{Y}}{\partial Z}+\left(6 H_{0} H_{X} H_{Y}+3 H_{0}^{2} H_{X Y}\right) \frac{\partial P_{0}}{\partial Z}\right] & P^{*}=P_{X X^{\prime}} \\
2 H_{X X}-\frac{\partial}{\partial \phi}\left(3 H_{0}^{2} H_{X} \frac{\partial P_{X^{\prime}}}{\partial \phi}\right)-\frac{\partial}{\partial Z}\left(3 H_{0}^{2} H_{X} \frac{\partial P_{X^{\prime}}}{\partial Z}\right) & P^{*}=P_{X Y^{\prime}} \\
2 H_{X Y}-\frac{\partial}{\partial \phi}\left(3 H_{0}^{2} H_{X} \frac{\partial P_{Y^{\prime}}}{\partial \phi}\right)-\frac{\partial}{\partial Z}\left(3 H_{0}^{2} H_{X} \frac{\partial P_{Y^{\prime}}}{\partial Z}\right) & P^{*}=P_{Y X^{\prime}} \\
2 H_{X Y}-\frac{\partial}{\partial \phi}\left(3 H_{0}^{2} H_{Y} \frac{\partial P_{X^{\prime}}}{\partial \phi}\right)-\frac{\partial}{\partial Z}\left(3 H_{0}^{2} H_{Y} \frac{\partial P_{X^{\prime}}}{\partial Z}\right) & P^{*}=P_{Y Y^{\prime}} \\
2 H_{Y Y}-\frac{\partial}{\partial \phi}\left(3 H_{0}^{2} H_{Y} \frac{\partial P_{Y^{\prime}}}{\partial \phi}\right)-\frac{\partial}{\partial Z}\left(3 H_{0}^{2} H_{Y} \frac{\partial P_{Y^{\prime}}}{\partial Z}\right) &
\end{array}
$$

$$
\begin{aligned}
& \frac{\partial}{\partial \phi}\left(H^{3} \frac{\partial P_{X X X}}{\partial \phi}\right)+\frac{\partial}{\partial Z}\left(H^{3} \frac{\partial P_{X X X}}{\partial Z}\right)= \\
& \frac{\partial H_{X X X}}{\partial \phi}-\frac{\partial}{\partial \phi}\left[9 H_{0}^{2} H_{X} \frac{\partial P_{X X}}{\partial \phi}+\left(18 H_{0} H_{X}^{2}+9 H_{0}^{2} H_{X X}\right) \frac{\partial P_{X}}{\partial \phi}\right. \\
& \left.+\left(6 H_{X}^{3}+18 H_{0} H_{X} H_{X X}+3 H_{0}^{2} H_{X X X}\right) \frac{\partial P_{0}}{\partial \phi}\right] \\
& -\frac{\partial}{\partial Z}\left[9 H_{0}^{2} H_{X} \frac{\partial P_{X X}}{\partial Z}+\left(18 H_{0} H_{X}^{2}+9 H_{0}^{2} H_{X X}\right) \frac{\partial P_{X}}{\partial Z}+\left(6 H_{X}^{3}+18 H_{0} H_{X} H_{X X}+3 H_{0}^{2} H_{X X X}\right) \frac{\partial P_{0}}{\partial Z}\right]
\end{aligned}
$$




$$
\begin{aligned}
& \frac{\partial}{\partial \phi}\left(H^{3} \frac{\partial P_{X X Y}}{\partial \phi}\right)+\frac{\partial}{\partial Z}\left(H^{3} \frac{\partial P_{X X Y}}{\partial Z}\right)= \\
& \frac{\partial H_{X X Y}}{\partial \phi}-\frac{\partial}{\partial \phi}\left[3 H_{0}^{2} H_{Y} \frac{\partial P_{X X}}{\partial \phi}+6 H_{0}^{2} H_{X} \frac{\partial P_{X Y}}{\partial \phi}+\left(12 H_{0} H_{X} H_{Y}+6 H_{0}^{2} H_{X Y}\right) \frac{\partial P_{X}}{\partial \phi}\right. \\
& \left.+\left(6 H_{0} H_{X}^{2}+3 H_{0}^{2} H_{X X}\right) \frac{\partial P_{Y}}{\partial \phi}+\left(6 H_{Y} H_{X}^{2}+12 H_{0} H_{X} H_{X Y}+6 H_{0} H_{Y} H_{X X}+3 H_{0}^{2} H_{X X Y}\right) \frac{\partial P_{0}}{\partial \phi}\right] \\
& -\frac{\partial}{\partial Z}\left[3 H_{0}^{2} H_{Y} \frac{\partial P_{X X}}{\partial Z}+6 H_{0}^{2} H_{X} \frac{\partial P_{X Y}}{\partial Z}+\left(12 H_{0} H_{X} H_{Y}+6 H_{0}^{2} H_{X Y}\right) \frac{\partial P_{X}}{\partial Z}\right. \\
& \left.+\left(6 H_{0} H_{X}^{2}+3 H_{0}^{2} H_{X X}\right) \frac{\partial P_{Y}}{\partial Z}+\left(6 H_{Y} H_{X}^{2}+12 H_{0} H_{X} H_{X Y}+6 H_{0} H_{Y} H_{X X}+3 H_{0}^{2} H_{X X Y}\right) \frac{\partial P_{0}}{\partial Z}\right]
\end{aligned}
$$

$$
\begin{aligned}
& \frac{\partial}{\partial \phi}\left(H^{3} \frac{\partial P_{X Y Y}}{\partial \phi}\right)+\frac{\partial}{\partial Z}\left(H^{3} \frac{\partial P_{X Y Y}}{\partial Z}\right)= \\
& \frac{\partial H_{X Y Y}}{\partial \phi}-\frac{\partial}{\partial \phi}\left[3 H_{0}^{2} H_{X} \frac{\partial P_{Y Y}}{\partial \phi}+6 H_{0}^{2} H_{Y} \frac{\partial P_{X Y}}{\partial \phi}+\left(12 H_{0} H_{X} H_{Y}+6 H_{0}^{2} H_{X Y}\right) \frac{\partial P_{Y}}{\partial \phi}\right. \\
& \left.+\left(6 H_{0} H_{Y}^{2}+3 H_{0}^{2} H_{Y Y}\right) \frac{\partial P_{X}}{\partial \phi}+\left(6 H_{X} H_{Y}^{2}+12 H_{0} H_{Y} H_{X Y}+6 H_{0} H_{X} H_{Y Y}+3 H_{0}^{2} H_{X Y Y}\right) \frac{\partial P_{0}}{\partial \phi}\right] \\
& -\frac{\partial}{\partial Z}\left[3 H_{0}^{2} H_{X} \frac{\partial P_{Y Y}}{\partial Z}+6 H_{0}^{2} H_{Y} \frac{\partial P_{X Y}}{\partial Z}+\left(12 H_{0} H_{X} H_{Y}+6 H_{0}^{2} H_{X Y}\right) \frac{\partial P_{Y}}{\partial Z}\right. \\
& \left.+\left(6 H_{0} H_{Y}^{2}+3 H_{0}^{2} H_{Y Y}\right) \frac{\partial P_{X}}{\partial Z}+\left(6 H_{X} H_{Y}^{2}+12 H_{0} H_{Y} H_{X Y}+6 H_{0} H_{X} H_{Y Y}+3 H_{0}^{2} H_{X Y Y}\right) \frac{\partial P_{0}}{\partial Z}\right]
\end{aligned}
$$




$$
\begin{aligned}
& \frac{\partial}{\partial \phi}\left(H^{3} \frac{\partial P_{Y Y Y}}{\partial \phi}\right)+\frac{\partial}{\partial Z}\left(H^{3} \frac{\partial P_{Y Y Y}}{\partial Z}\right)= \\
& \frac{\partial H_{Y Y Y}}{\partial \phi}-\frac{\partial}{\partial \phi}\left[9 H_{0}^{2} H_{Y} \frac{\partial P_{Y Y}}{\partial \phi}+\left(18 H_{0} H_{Y}^{2}+9 H_{0}^{2} H_{Y Y}\right) \frac{\partial P_{Y}}{\partial \phi}\right. \\
& \left.+\left(6 H_{Y}^{3}+18 H_{0} H_{Y} H_{Y Y}+3 H_{0}^{2} H_{Y Y Y}\right) \frac{\partial P_{0}}{\partial \phi}\right] \\
& -\frac{\partial}{\partial Z}\left[9 H_{0}^{2} H_{Y} \frac{\partial P_{Y Y}}{\partial Z}+\left(18 H_{0} H_{Y}^{2}+9 H_{0}^{2} H_{Y Y}\right) \frac{\partial P_{Y}}{\partial Z}+\left(6 H_{Y}^{3}+18 H_{0} H_{Y} H_{Y Y}+3 H_{0}^{2} H_{Y Y Y}\right) \frac{\partial P_{0}}{\partial Z}\right]
\end{aligned}
$$

$$
\begin{aligned}
& \frac{\partial}{\partial \phi}\left(H^{3} \frac{\partial P_{X X X^{\prime}}}{\partial \phi}\right)+\frac{\partial}{\partial Z}\left(H^{3} \frac{\partial P_{X X X^{\prime}}}{\partial Z}\right)= \\
& 2 H_{X X X}-\frac{\partial}{\partial \phi}\left[6 H_{0}^{2} H_{X} \frac{\partial P_{X X^{\prime}}}{\partial \phi}+\left(6 H_{0} H_{X}^{2}+3 H_{0}^{2} H_{X X}\right) \frac{\partial P_{X^{\prime}}}{\partial \phi}\right] \\
& -\frac{\partial}{\partial Z}\left[6 H_{0}^{2} H_{X} \frac{\partial P_{X X^{\prime}}}{\partial Z}+\left(6 H_{0} H_{X}^{2}+3 H_{0}^{2} H_{X X}\right) \frac{\partial P_{X^{\prime}}}{\partial Z}\right]
\end{aligned}
$$

$$
\begin{aligned}
& \frac{\partial}{\partial \phi}\left(H^{3} \frac{\partial P_{X X Y^{\prime}}}{\partial \phi}\right)+\frac{\partial}{\partial Z}\left(H^{3} \frac{\partial P_{X X Y^{\prime}}}{\partial Z}\right)= \\
& 2 H_{X X Y}-\frac{\partial}{\partial \phi}\left[6 H_{0}^{2} H_{X} \frac{\partial P_{X Y^{\prime}}}{\partial \phi}+\left(6 H_{0} H_{X}^{2}+3 H_{0}^{2} H_{X X}\right) \frac{\partial P_{Y^{\prime}}}{\partial \phi}\right] \\
& -\frac{\partial}{\partial Z}\left[6 H_{0}^{2} H_{X} \frac{\partial P_{X Y^{\prime}}}{\partial Z}+\left(6 H_{0} H_{X}^{2}+3 H_{0}^{2} H_{X X}\right) \frac{\partial P_{Y^{\prime}}}{\partial Z}\right]
\end{aligned}
$$

$$
\begin{aligned}
& \frac{\partial}{\partial \phi}\left(H^{3} \frac{\partial P_{Y Y X^{\prime}}}{\partial \phi}\right)+\frac{\partial}{\partial Z}\left(H^{3} \frac{\partial P_{Y Y X^{\prime}}}{\partial Z}\right)= \\
& 2 H_{X Y Y}-\frac{\partial}{\partial \phi}\left[6 H_{0}^{2} H_{Y} \frac{\partial P_{Y X^{\prime}}}{\partial \phi}+\left(6 H_{0} H_{Y}^{2}+3 H_{0}^{2} H_{Y Y}\right) \frac{\partial P_{X^{\prime}}}{\partial \phi}\right] \\
& -\frac{\partial}{\partial Z}\left[6 H_{0}^{2} H_{Y} \frac{\partial P_{Y X^{\prime}}}{\partial Z}+\left(6 H_{0} H_{Y}^{2}+3 H_{0}^{2} H_{Y Y}\right) \frac{\partial P_{X^{\prime}}}{\partial Z}\right]
\end{aligned}
$$




$$
\begin{aligned}
& \frac{\partial}{\partial \phi}\left(H^{3} \frac{\partial P_{Y Y Y^{\prime}}}{\partial \phi}\right)+\frac{\partial}{\partial Z}\left(H^{3} \frac{\partial P_{Y Y Y^{\prime}}}{\partial Z}\right)= \\
& 2 H_{Y Y Y}-\frac{\partial}{\partial \phi}\left[6 H_{0}^{2} H_{Y} \frac{\partial P_{Y Y^{\prime}}}{\partial \phi}+\left(6 H_{0} H_{Y}^{2}+3 H_{0}^{2} H_{Y Y}\right) \frac{\partial P_{Y^{\prime}}}{\partial \phi}\right] \\
& -\frac{\partial}{\partial Z}\left[6 H_{0}^{2} H_{Y} \frac{\partial P_{Y Y^{\prime}}}{\partial Z}+\left(6 H_{0} H_{Y}^{2}+3 H_{0}^{2} H_{Y Y}\right) \frac{\partial P_{Y^{\prime}}}{\partial Z}\right]
\end{aligned}
$$

$$
\begin{aligned}
& \frac{\partial}{\partial \phi}\left(H^{3} \frac{\partial P_{X Y X^{\prime}}}{\partial \phi}\right)+\frac{\partial}{\partial Z}\left(H^{3} \frac{\partial P_{X Y X^{\prime}}}{\partial Z}\right)= \\
& 2 H_{X X Y}-\frac{\partial}{\partial \phi}\left[3 H_{0}^{2} H_{Y} \frac{\partial P_{X X^{\prime}}}{\partial \phi}+3 H_{0}^{2} H_{X} \frac{\partial P_{Y X^{\prime}}}{\partial \phi}+\left(6 H_{0} H_{X} H_{Y}+3 H_{0}^{2} H_{X Y}\right) \frac{\partial P_{X^{\prime}}}{\partial \phi}\right] \\
& -\frac{\partial}{\partial Z}\left[3 H_{0}^{2} H_{Y} \frac{\partial P_{X X^{\prime}}}{\partial Z}+3 H_{0}^{2} H_{X} \frac{\partial P_{Y X^{\prime}}}{\partial Z}+\left(6 H_{0} H_{X} H_{Y}+3 H_{0}^{2} H_{X Y}\right) \frac{\partial P_{X^{\prime}}}{\partial Z}\right]
\end{aligned}
$$

$$
\begin{aligned}
& \frac{\partial}{\partial \phi}\left(H^{3} \frac{\partial P_{X Y Y^{\prime}}}{\partial \phi}\right)+\frac{\partial}{\partial Z}\left(H^{3} \frac{\partial P_{X Y Y^{\prime}}}{\partial Z}\right)= \\
& 2 H_{X Y Y}-\frac{\partial}{\partial \phi}\left[3 H_{0}^{2} H_{Y} \frac{\partial P_{X Y^{\prime}}}{\partial \phi}+3 H_{0}^{2} H_{X} \frac{\partial P_{Y Y^{\prime}}}{\partial \phi}+\left(6 H_{0} H_{X} H_{Y}+3 H_{0}^{2} H_{X Y}\right) \frac{\partial P_{Y^{\prime}}}{\partial \phi}\right] \\
& -\frac{\partial}{\partial Z}\left[3 H_{0}^{2} H_{Y} \frac{\partial P_{X Y^{\prime}}}{\partial Z}+3 H_{0}^{2} H_{X} \frac{\partial P_{Y Y^{\prime}}}{\partial Z}+\left(6 H_{0} H_{X} H_{Y}+3 H_{0}^{2} H_{X Y}\right) \frac{\partial P_{Y^{\prime}}}{\partial Z}\right]
\end{aligned}
$$

\subsection{Nonlinear bearing forces}

Expanding the dimensionless bearing forces $\bar{F}_{X}$ and $\bar{F}_{Y}$ to the third order using Taylor series, for which $\bar{F}_{X}=\frac{F_{x}}{W}$ and $\bar{F}_{Y}=\frac{F_{y}}{W}$, where $F_{x}$ is the bearing force in $x$ direction, $F_{y}$ is 
the bearing force in $y$ direction and $W$ is the bearing load.

$$
\begin{aligned}
\bar{F}_{\zeta}= & \bar{F}_{\zeta_{0}}+\frac{\partial \bar{F}_{\zeta}}{\partial X} \delta X+\frac{\partial \bar{F}_{\zeta}}{\partial Y} \delta Y+\frac{\partial \bar{F}_{\zeta}}{\partial X^{\prime}} \delta X^{\prime}+\frac{\partial \bar{F}_{\zeta}}{\partial Y^{\prime}} \delta Y^{\prime}+0.5 \frac{\partial^{2} \bar{F}_{\zeta}}{\partial X^{2}} \delta X^{2}+ \\
& 0.5 \frac{\partial^{2} \bar{F}_{\zeta}}{\partial Y^{2}} \delta Y^{2}+\frac{\partial^{2} \bar{F}_{\zeta}}{\partial X \partial Y} \delta X \delta Y+\frac{\partial^{2} \bar{F}_{\zeta}}{\partial X \partial X^{\prime}} \delta X \delta X^{\prime}+\frac{\partial^{2} \bar{F}_{\zeta}}{\partial X \partial Y^{\prime}} \delta X \delta Y^{\prime}+\frac{\partial^{2} \bar{F}_{\zeta}}{\partial Y \partial X^{\prime}} \delta Y \delta X^{\prime}+ \\
& \frac{\partial^{2} \bar{F}_{\zeta}}{\partial Y \partial Y^{\prime}} \delta Y \delta Y^{\prime}+\frac{1}{6} \frac{\partial^{3} \bar{F}_{\zeta}}{\partial X^{3}} \delta X^{3}+\frac{1}{6} \frac{\partial^{3} \bar{F}_{\zeta}}{\partial Y^{3}} \delta Y^{3}+0.5 \frac{\partial^{3} \bar{F}_{\zeta}}{\partial X^{2} \partial Y^{2}} \delta X^{2} \delta Y+ \\
& 0.5 \frac{\partial^{3} \bar{F}_{\zeta}}{\partial X \partial Y^{2}} \delta X \delta Y^{2}+0.5 \frac{\partial^{3} \bar{F}_{\zeta}}{\partial X^{2} \partial X^{\prime}} \delta X^{2} \delta X^{\prime}+0.5 \frac{\partial^{3} \bar{F}_{\zeta}}{\partial X^{2} \partial Y^{\prime}} \delta X^{2} \delta Y^{\prime}+ \\
& 0.5 \frac{\partial^{3} \bar{F}_{\zeta}}{\partial Y^{2} \partial X^{\prime}} \delta Y^{2} \delta X^{\prime}+0.5 \frac{\partial^{3} \bar{F}_{\zeta}}{\partial Y^{2} \partial Y^{\prime}} \delta Y^{2} \delta Y^{\prime}+\frac{\partial^{3} \bar{F}_{\zeta}}{\partial X \partial Y \partial X^{\prime}} \delta X \delta Y \delta X^{\prime}+\frac{\partial^{3} \bar{F}_{\zeta}}{\partial X \partial Y \partial Y^{\prime}} \delta X \delta Y \delta Y^{\prime}
\end{aligned}
$$

where $\zeta=X, Y$.

The bearing forces in $X, Y$ directions in Eq. (31) can be written using the bearing first, second and third order coefficients as shown below:

$$
\begin{aligned}
\bar{F}_{\zeta}= & \bar{F}_{\zeta_{0}}+K_{\zeta X} \delta X+K_{\zeta Y} \delta Y+C_{\zeta X} \delta X^{\prime}+C_{\zeta Y} \delta Y^{\prime}+0.5 K_{\zeta X X} \delta X^{2}+ \\
& 0.5 K_{\zeta Y Y} \delta Y^{2}+K_{\zeta X Y} \delta X \delta Y+C_{\zeta X X} \delta X \delta X^{\prime}+C_{\zeta X Y} \delta X \delta Y^{\prime}+C_{\zeta Y X} \delta Y \delta X^{\prime}+ \\
& C_{\zeta Y Y} \delta Y \delta Y^{\prime}+\frac{1}{6} K_{\zeta X X X} \delta X^{3}+\frac{1}{6} K_{\zeta Y Y Y} \delta Y^{3}+0.5 K_{\zeta X X Y} \delta X^{2} \delta Y+ \\
& 0.5 K_{\zeta X Y Y} \delta X \delta Y^{2}+0.5 C_{\zeta X X X} \delta X^{2} \delta X^{\prime}+0.5 C_{\zeta X X Y} \delta X^{2} \delta Y^{\prime}+ \\
& 0.5 C_{\zeta Y Y X} \delta Y^{2} \delta X^{\prime}+0.5 C_{\zeta Y Y Y} \delta Y^{2} \delta Y^{\prime}+C_{\zeta X Y X} \delta X \delta Y \delta X^{\prime}+C_{\zeta X Y Y} \delta X \delta Y \delta Y^{\prime}
\end{aligned}
$$

for which $X=\frac{x}{c}$ and $Y=\frac{Y}{c}$ are the dimensionless coordinates, $c$ is the radial clearance. $K_{i j}=\frac{k_{i j} c}{W}$ and $C_{i j}=\frac{c_{i j} c \Omega}{W}$ are the dimensionless linear stiffness and damping coefficients respectively, where $(i, j=X, Y) . k_{i j}$ and $c_{i j}$ are stiffness and damping dimensional coefficients respectively.

$$
K_{i j k}=\frac{k_{i j k} c^{2}}{W} \text { and } C_{i j k}=\frac{C_{i j k} c^{2} \Omega}{W} \text { are the second order dimensionless stiffness and damp- }
$$
ing coefficients respectively where $i, j, k=X, Y . k_{i j k}$ and $c_{i j k}$ are the second order stiffness 
and damping dimensional coefficients respectively.

$K_{i j k l}=\frac{k_{i j k l} c^{3}}{W}$ and $C_{i j k l}=\frac{C_{i j k l} c^{3} \Omega}{W}$ are the third order dimensionless stiffness and damping coefficients where $i, j, k, l=X, Y . k_{i j k l}$ and $c_{i j k l}$ are the third order stiffness and damping dimensional coefficients.

The bearing forces can be calculated by integrating the pressure over the area as shown below

$$
\begin{aligned}
& \bar{F}_{X}=\int_{0}^{\frac{L}{R}} \int_{0}^{2 \pi}-P \sin \phi d \phi d Z \\
& \bar{F}_{Y}=\int_{0}^{\frac{L}{R}} \int_{0}^{2 \pi}-P \cos \phi d \phi d Z
\end{aligned}
$$

Comparing Eqs. (18), 32) to (34) the steady state force can be obtained as:

$$
\begin{aligned}
\bar{F}_{X_{0}} & =\int_{0}^{\frac{L}{R}} \int_{0}^{2 \pi}-P_{0} \sin \phi d \phi d Z \\
\bar{F}_{Y_{0}} & =\int_{0}^{\frac{L}{R}} \int_{0}^{2 \pi}-P_{0} \cos \phi d \phi d Z
\end{aligned}
$$

It is worth noting that through the present analysis the only steady state load in $y$ direction is the weight and no static load in $x$ direction. Therefore, $F_{x 0}=0$ and $F_{y 0}=W$ which also means that

$$
\bar{F}_{X_{0}}=0, \bar{F}_{Y_{0}}=1
$$

Using this condition and by an iterative method the steady state attitude angle can be obtained. Also, the eight first order coefficients can be obtained from the following equations. 


$$
\begin{aligned}
K_{X \zeta} & =\int_{0}^{\frac{L}{R}} \int_{0}^{2 \pi}-P_{\zeta} \sin \phi \mathrm{d} \phi \mathrm{d} Z \\
K_{Y \zeta} & =\int_{0}^{\frac{L}{R}} \int_{0}^{2 \pi}-P_{\zeta} \cos \phi \mathrm{d} \phi \mathrm{d} Z \\
C_{X \zeta} & =\int_{0}^{\frac{L}{R}} \int_{0}^{2 \pi}-P_{\zeta^{\prime}} \sin \phi \mathrm{d} \phi \mathrm{d} Z \\
C_{Y \zeta} & =\int_{0}^{\frac{L}{R}} \int_{0}^{2 \pi}-P_{\zeta^{\prime}} \cos \phi \mathrm{d} \phi \mathrm{d} Z
\end{aligned}
$$

where $\zeta=X, Y$ which means that by replacing $\zeta$ by either $X$ or $Y$ the eight equation for the eight coefficients can be obtained from Eqs.(38) to (41) In addition, the fourteen second order coefficients can be calculated from

$$
\begin{aligned}
K_{X \zeta \eta} & =\int_{0}^{\frac{L}{R}} \int_{0}^{2 \pi}-P_{\zeta \eta} \sin \phi \mathrm{d} \phi \mathrm{d} Z \\
K_{Y \zeta \eta} & =\int_{0}^{\frac{L}{R}} \int_{0}^{2 \pi}-P_{\zeta \eta} \cos \phi \mathrm{d} \phi \mathrm{d} Z \\
C_{X \zeta \eta} & =\int_{0}^{\frac{L}{R}} \int_{0}^{2 \pi}-P_{\zeta \eta^{\prime}} \sin \phi \mathrm{d} \phi \mathrm{d} Z \\
C_{Y \zeta \eta} & =\int_{0}^{\frac{L}{R}} \int_{0}^{2 \pi}-P_{\zeta \eta^{\prime}} \cos \phi \mathrm{d} \phi \mathrm{d} Z
\end{aligned}
$$

where each of $\zeta$ and $\eta$ can be either $X, Y$. This means that Eqs. 42 to 45 represent sixteen equations. These equations are reduced to fourteen equations because $K_{X Y X}=K_{X X Y}$ and $K_{Y X Y}=K_{Y Y X}$. Similarly, the twenty Third order coefficients can be evaluated from the following equations 


$$
\begin{aligned}
K_{X \zeta \eta \gamma} & =\int_{0}^{\frac{L}{R}} \int_{0}^{2 \pi}-P_{\zeta \eta \gamma} \sin \phi \mathrm{d} \phi \mathrm{d} Z \\
K_{Y \zeta \eta \gamma} & =\int_{0}^{\frac{L}{R}} \int_{0}^{2 \pi}-P_{\zeta \eta \gamma} \cos \phi \mathrm{d} \phi \mathrm{d} Z \\
C_{X \zeta \eta \gamma} & =\int_{0}^{\frac{L}{R}} \int_{0}^{2 \pi}-P_{\zeta \eta \gamma^{\prime}} \sin \phi \mathrm{d} \phi \mathrm{d} Z \\
C_{Y \zeta \eta \gamma} & =\int_{0}^{\frac{L}{R}} \int_{0}^{2 \pi}-P_{\zeta \eta \gamma^{\prime}} \cos \phi \mathrm{d} \phi \mathrm{d} Z
\end{aligned}
$$

where each of $\zeta, \eta$ and $\gamma$ can be either $X, Y$. This means that thirty-two equation can be obtained from Eqs. (46) to (49) but these equations are reduced to only twenty because of similarity of several coefficients such as $K_{X Y X X}=K_{X X Y X}=K_{X X X Y}$.

\subsection{Rotor-bearing model}

In this section, the analytical model for elastic rotor supported on two symmetric journal bearings is investigated. The model is four degree of freedom in $x$ and $y$ directions. In this model both the mass of rotor inside the journal $m_{j}$ and disk mass $m_{d}$ are considered, as shown in Figure 2, A static load $2 W$ is applied on the disc. 
(a)

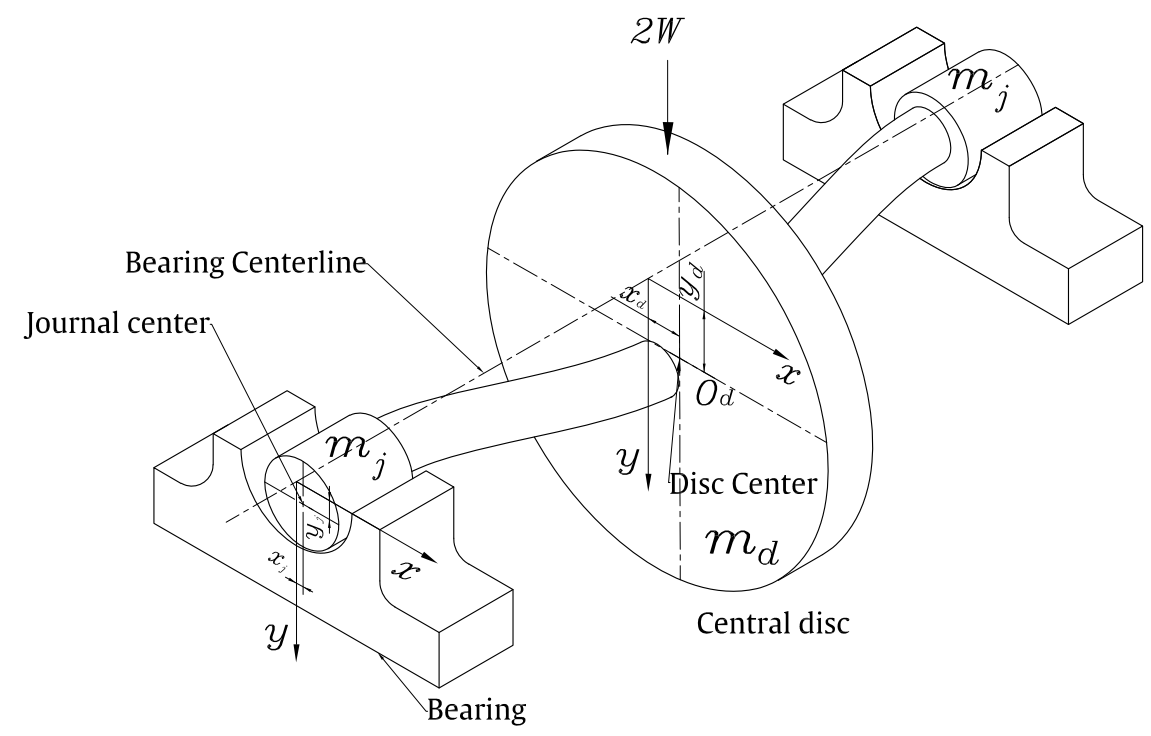

(b)

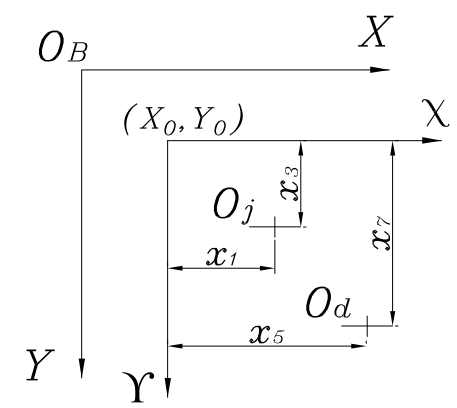

Figure 2: (a) Elastic rotor supported on two symmetric journal bearings. (b) Bearing coordinates.

The equations of motion for the presented system can be presented as

$$
\begin{aligned}
2 m_{j} \ddot{x}_{j}+k_{s}\left(x_{j}-x_{d}\right) & =-2 F_{x} \\
2 m_{j} \ddot{y}_{j}+k_{s}\left(y_{j}-y_{d}\right) & =-2 F_{y} \\
m_{d} \ddot{x}_{d}+k_{s}\left(x_{d}-x_{j}\right) & =0 \\
m_{d} \ddot{y}_{d}+k_{s}\left(y_{d}-y_{j}\right) & =2 W
\end{aligned}
$$

For which $m_{j}$ is the mass of shaft inside and around the journal and $m_{d}$ is the sum of the disc and remaining part of the shaft mass, $k_{s}$ is the shaft stiffness, $F_{x}$ and $F_{y}$ are the bearing forces in $x$ and $y$ directions respectively, $W$ is the bearing load and $x_{j}, y_{j}, x_{d}, y_{d}$ are journal mass geometrical center and disc geometrical center current positions with respect to the journal steady state position. $m=m_{d}+2 m_{j}$ is the total mass of the rotor.

Transforming the equations of motion Eq. (50) into dimensionless form we get the follow- 
ing equation

$$
\begin{aligned}
2 M_{J} X_{J}^{\prime \prime}+K_{S}\left(X_{J}-X_{D}\right) & =-2 \bar{F}_{x} \\
2 M_{J} Y_{J}^{\prime \prime}+K_{S}\left(Y_{J}-Y_{D}\right) & =-2 \bar{F}_{y} \\
M_{D} X_{D}^{\prime \prime}+K_{S}\left(X_{D}-X_{J}\right) & =0 \\
M_{D} Y_{D}^{\prime \prime}+K_{S}\left(Y_{D}-Y_{J}\right) & =2
\end{aligned}
$$

where

$$
\begin{aligned}
& X_{J}=\frac{x_{j}}{c}, Y_{J}=\frac{y_{j}}{c}, X_{D}=\frac{x_{D}}{c}, Y_{D}=\frac{y_{D}}{c},{ }^{\prime}=\frac{\mathrm{d}}{\mathrm{d} \tau},{ }^{\prime \prime}=\frac{\mathrm{d}^{2}}{\mathrm{~d} \tau^{2}}, \tau=\Omega t \\
& X_{J}^{\prime \prime}=\frac{\ddot{x}_{j}}{\Omega^{2} c}, Y_{J}=\frac{\ddot{y}_{j}}{\Omega^{2} c}, X_{D}^{\prime \prime}=\frac{\ddot{x}_{d}}{\Omega^{2} c}, Y_{D}^{\prime \prime}=\frac{\ddot{y}_{d}}{\Omega^{2} c}, K_{S}=\frac{k_{s} c}{W}, M_{J}=\frac{m_{j} \Omega^{2} c}{W} \text { and } M_{D}=\frac{m_{d} \Omega^{2} c}{W} .
\end{aligned}
$$

$M_{J}$ and $M_{D}$ are the dimensionless mass of the journal and disc respectively. Through the current paper the following mass ratio are considered $2 M_{J}=0.1 M$ and $M_{D}=0.9 M$. The dimensionless shaft stiffness is $K_{S}$. The rotational speed is $\Omega$ and the dimensionless time is $\tau$. The dimensionless static equilibrium forces are $\bar{F}_{x_{0}}=0$ and $\bar{F}_{y_{0}}=1$. Substituting Eqs.(32) and(37) in Eq.(51), and converting the resulting equations into in the state space 
form, the final resulting equations can be written as:

$$
x_{2}^{\prime}=\frac{K_{s}\left(x_{5}-x_{1}\right)}{2 M_{J}}-\frac{1}{M_{J}}\left(\begin{array}{c}
x_{2} \\
+0.5 K_{X Y Y} x_{3}^{2}+K_{X X Y} x_{1} x_{3}+C_{X X X} x_{1} x_{2}+C_{X X Y} x_{1} x_{4} \\
+C_{X Y X} x_{3} x_{2}+C_{X Y Y} x_{3} x_{4}+\frac{1}{6} K_{X X X X} x_{1}^{3}+\frac{1}{6} K_{X Y Y Y} x_{3}^{3} \\
+0.5 K_{X X X Y} x_{1}^{2} x_{3}+0.5 K_{X X Y Y} x_{1} x_{3}^{2}+0.5 C_{X X X X} x_{1}^{2} x_{2}+ \\
0.5 C_{X X X Y} x_{1}^{2} x_{4}+0.5 C_{X Y Y X} x_{3}^{2} x_{2}+0.5 C_{X Y Y Y} x_{3}^{2} x_{4}+ \\
C_{X X Y X} x_{1} x_{3} x_{2}+C_{X X Y Y} x_{1} x_{3} x_{4}
\end{array}\right)
$$$$
x_{3}^{\prime}=
$$

$x_{4}$

$$
x_{4}^{\prime}=\frac{K_{s}\left(x_{7}-x_{3}\right)}{2 M_{J}}-\frac{1}{M_{J}}\left(\begin{array}{l}
1+K_{Y X} x_{1}+C_{Y X} x_{2}+K_{Y Y} x_{3}+C_{Y Y} x_{4}+0.5 K_{Y X X} x_{1}^{2} \\
+0.5 K_{Y Y Y} x_{3}^{2}+K_{Y X Y} x_{1} x_{3}+C_{Y X X} x_{1} x_{2}+C_{Y X Y} x_{1} x_{4} \\
+C_{Y Y X} x_{3} x_{2}+C_{Y Y Y} x_{3} x_{4}+\frac{1}{6} K_{Y X X X} x_{1}^{3}+\frac{1}{6} K_{Y Y Y Y} x_{3}^{3} \\
+0.5 K_{Y X X Y} x_{1}^{2} x_{3}+0.5 K_{Y X Y Y} x_{1} x_{3}^{2}+0.5 C_{Y X X X} x_{1}^{2} x_{2} \\
+0.5 C_{Y X X Y} x_{1}^{2} x_{4}+0.5 C_{Y Y Y X} x_{3}^{2} x_{2}+0.5 C_{Y Y Y Y} x_{3}^{2} x_{4}+ \\
C_{Y X Y X} x_{1} x_{3} x_{2}+C_{Y X Y Y} x_{1} x_{3} x_{4}
\end{array}\right)
$$

$x_{5}^{\prime}=$

$x_{6}^{\prime}=$

$x_{7}^{\prime}=$

$x_{8}^{\prime}=$ $x_{6}$

\section{$\frac{K_{s}\left(x_{1}-x_{5}\right)}{M_{D}}$}

$x_{8}$

$\frac{2+K_{s}\left(x_{3}-x_{7}\right)}{M_{D}}$

where $x_{1}=X_{J}, x_{2}=X_{J}^{\prime}, x_{3}=Y_{J}, x_{4}=Y_{J}^{\prime}, x_{5}=X_{D}, x_{6}=X_{D}^{\prime}, x_{7}=Y_{D}, x_{8}=Y_{D}^{\prime}$. 
Finally, Eq.52 can be written as

$$
\mathbf{x}^{\prime}=\mathbf{f}(\mathbf{x}, \bar{M})
$$

where $\mathbf{x}=\left[\begin{array}{llllllll}x_{1} & x_{2} & x_{3} & x_{4} & x_{5} & x_{5} & x_{7} & x_{8}\end{array}\right], \bar{M}=M_{D}+2 M_{J}$ and $\frac{M_{J}}{M_{D}}=\frac{1}{18}$.

The system of equations in Eq. (53) can have a periodic solution $x(t)=x(t+T)$. This is occurred above what is called the threshold speed $\left(\bar{M} \geq \bar{M}_{t h}\right)$, where $T$ is the periodic time of solution and $\bar{M}_{t h}$ is the dimensionless threshold mass of the system. This will be discussed in detailed in the results section.

\section{Results and discussion}

In this section, the first, second and third order bearing coefficients are calculated using infinitesimal perturbation analysis. The bearing coefficients are presented and listed for full circular journal bearing. Perturbation analysis is used to investigate the validity range of these coefficients. Afterwards, the bearing coefficients are used to investigate the dynamics of flexible rotor supported by two symmetric journal bearings.

\subsection{Journal bearing coefficients and mesh sensitivity}

In this section, the bearing linear and nonlinear coefficients based on first, second and third order coefficients are evaluated. This is done using infinitesimal perturbation as discussed in analysis section. The presented results are for un-grooved full circular journal bearing with $L / D$ ratio of 1 . The linear bearing coefficients are eight and plotted in Figure 3 a. The nonlinear second order bearing coefficients are fourteen and are plotted in Figure $3 \mathrm{~b}$. The third order coefficients are twenty and are plotted in Figure 4. The effect of mesh size on the bearing coefficients is also investigated for all the coefficients. Several mesh sizes are investigated but only four cases are presented to avoid over density of the figures. The presented mesh sizes are selected to vary from coarse to fine mesh. These mesh sizes are 
$50 \times 100,100 \times 200,150 \times 600$ and $200 \times 800$. In both Figure 3 and Figure 4 the color is used to differentiate the bearing coefficients and the line type is used to differentiate the mesh size as shown in the subfigures legend and the figure general legend. The results of Figure 3 show that for the plotted coefficients the results of mesh $100 \times 400,150 \times 600$ and $200 \times 800$ are identical over the full range of Sommerfeld number. The results of Figure 4 show that the bearing coefficients obtained using mesh $100 \times 400$ converge with that $200 \times 800$ in most coefficients. However, for $K_{X X X X}, C_{X X X X}, K_{Y X X X}$ and $C_{Y X X X}$ mesh $100 \times 400$ is not sufficient. In addition, the figure shows that mesh $150 \times 600$ and $200 \times 800$ results are converged for all coefficients. Therefore, mesh $150 \times 600$ was considered as minimum mesh used in coefficients evaluation during the present paper. Numerical values or the obtained parameters using mesh size of $150 \times 600$ are presented in Table B.1 and Table B.2. 
(a)
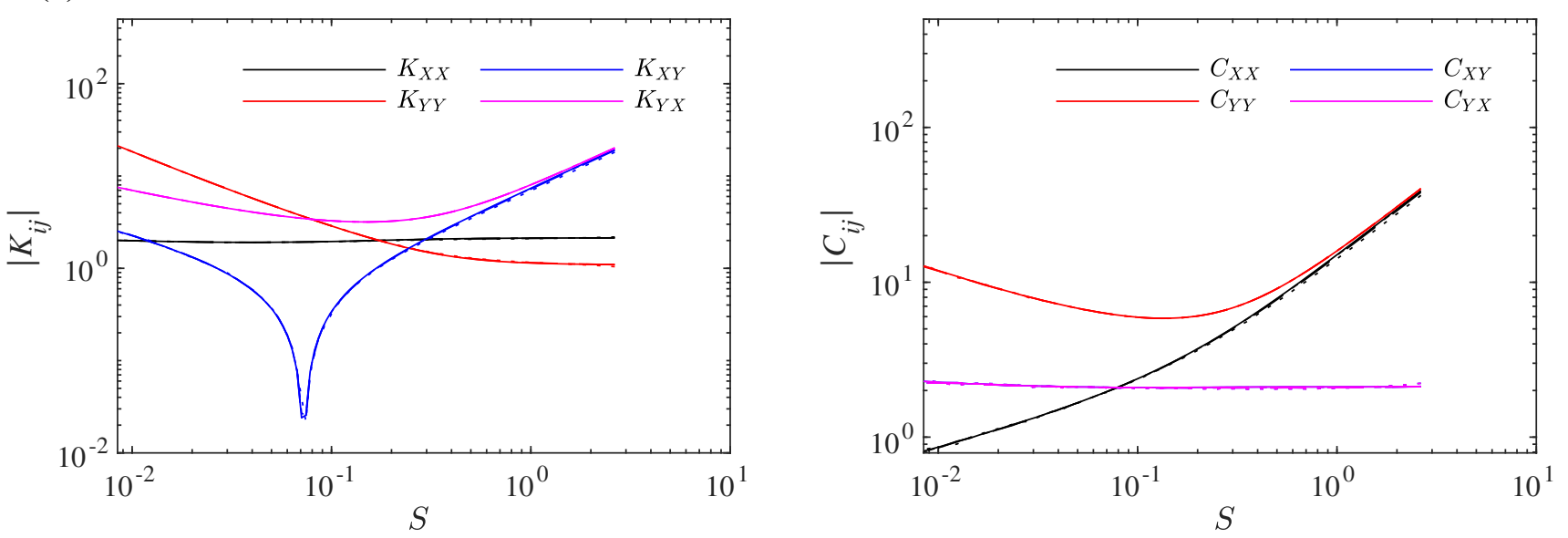

(b)
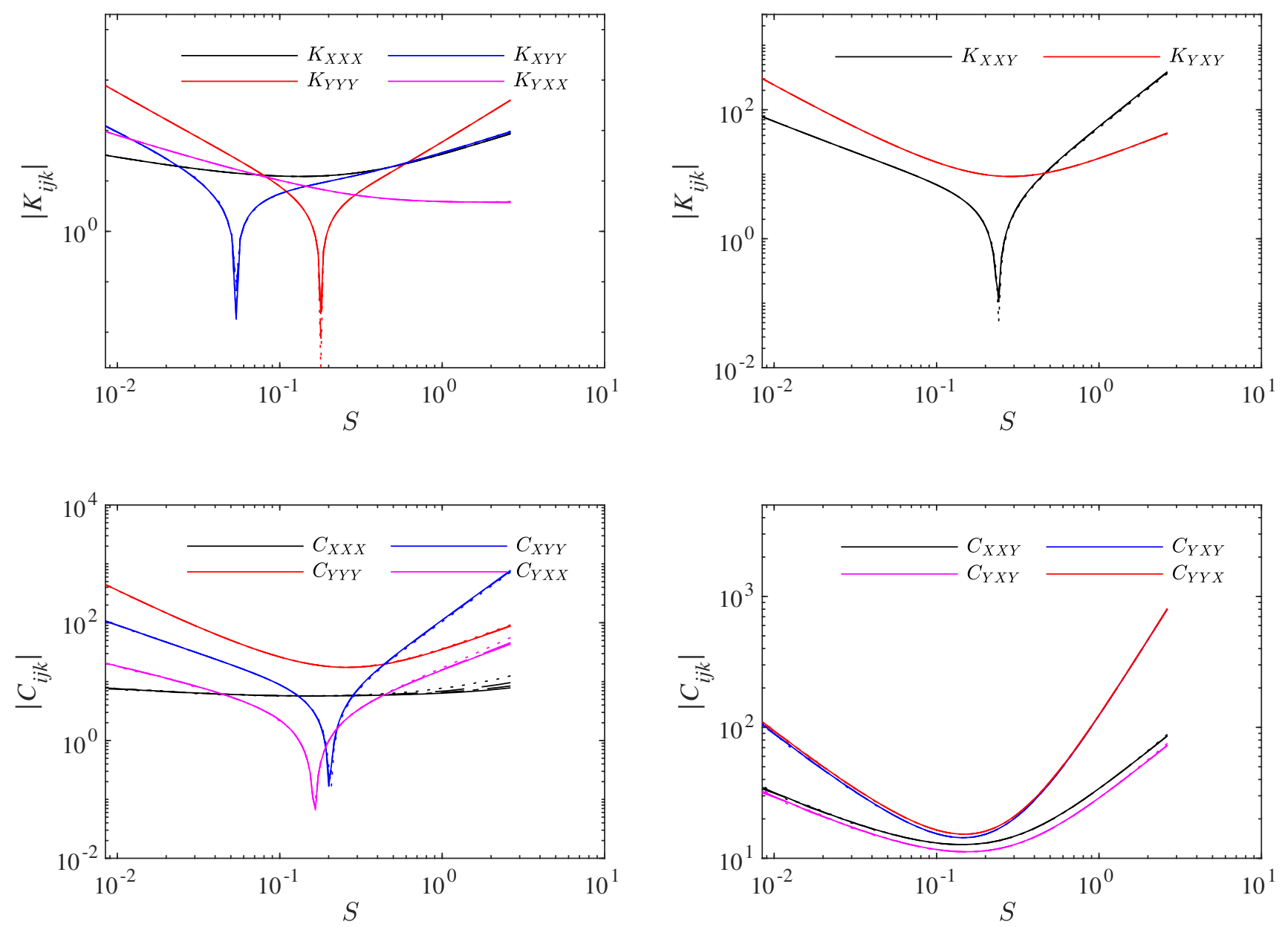

Figure 3: (Color online) Loglog plot for un-grooved journal bearing stiffness and damping coefficients(absolute) versus the Sommerfeld number. $L / D=1$, (a) First order bearing coefficient (b) Second order bearing coefficient. In this figure the dotted line for mesh $50 \times 200$, dashed line for mesh $100 \times 400$, centerline for mesh $150 \times 600$ and solid line for mesh $200 \times 800$. 

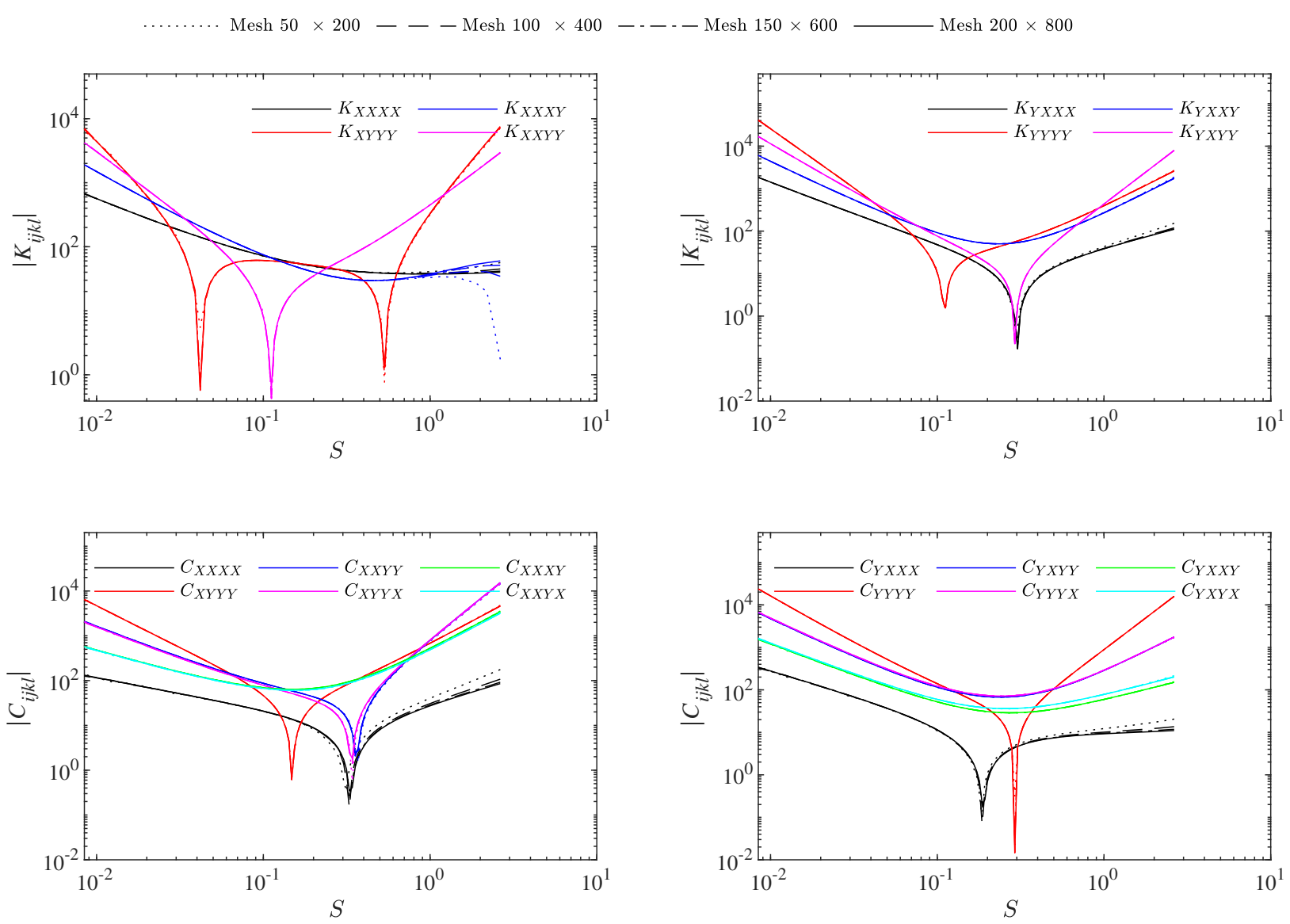

Figure 4: (Color online) Loglog plot for un-grooved full circular journal bearing third order stiffness and damping coefficients (absolute) versus the Sommerfeld number. $L / D$ ratio is unity. In this figure the dotted line for mesh $50 \times 200$, dashed line for mesh $100 \times 400$, centerline for mesh $150 \times 600$ and solid line for mesh $200 \times 800$

\subsection{Perturbation Analysis}

In this section, a perturbation analysis is used to validate the obtained bearing coefficients. This analysis is executed using un-grooved full circular journal bearing with $L / D$ ratio of 1 . This is done by applying displacement and velocity perturbations to the equilibrium point then evaluate the obtained forces using four different methods. These methods are based on direct integration of Reynolds equation $\left(\bar{F}_{R e}\right)$, first order coefficients $\left(\bar{F}_{1}\right)$, second order coefficients $\left(\bar{F}_{2}\right)$ and third order coefficients $\left(\bar{F}_{3}\right)$. The component of these forces in $X$ and $Y$ directions are evaluated and plotted in Figure 5. To investigate the 
validity range of the bearing linear and nonlinear coefficients in evaluating the nonlinear bearing forces around the equilibrium point, four different values of perturbations are used. The first one is $\left(\delta X=0.0001, \delta X^{\prime}=0.0001, \delta Y=0.0001\right.$ and $\left.\delta Y^{\prime}=0.0001\right)$ and its results are plotted in Figure 5 a. In Figure 5 the left column represents the $X$ component of the perturbation force and the right column represent the $Y$ component of the perturbation force. All the subfigures of Figure 5 are drawn versus the Sommerfeld number. Figure $5 \mathrm{~b}, \mathrm{c}$ and $\mathrm{d}$ are for perturbations $\left(\delta X=0.001, \delta X^{\prime}=0.001, \delta Y=0.001\right.$ and $\left.\delta Y^{\prime}=0.001\right)$, $\left(\delta X=0.01, \delta X^{\prime}=0.01, \delta Y=0.01\right.$ and $\left.\delta Y^{\prime}=0.01\right)$ and $\left(\delta X=0.1, \delta X^{\prime}=0.1, \delta Y=0.1\right.$ and $\left.\delta Y^{\prime}=0.1\right)$ respectively. The figure results show that at low perturbations the forces evaluated using all the four methods are approximately the same as shown in Figure 5 a, b. However, by increasing the values of perturbation as in Figure 5-c, a significant deviation between the forces evaluated based on the nonlinear coefficients and that based on the direct Reynolds integration forces $\left(\bar{F}_{R e}\right)$ is recognized. The last case Figure 5-d shows that at very low and high Sommerfeld number the deviation between the forces is larger than when $S \in[0.1-0.6]$. Also, the $x$ component of force approximation based on the third order coefficients was closer to the forces based on direct integration of Reynolds equation at $S<0.55$ and $y$ component of force approximation was closer to the forces based on direct integration of Reynolds equation when $S<0.3$. 
(a)
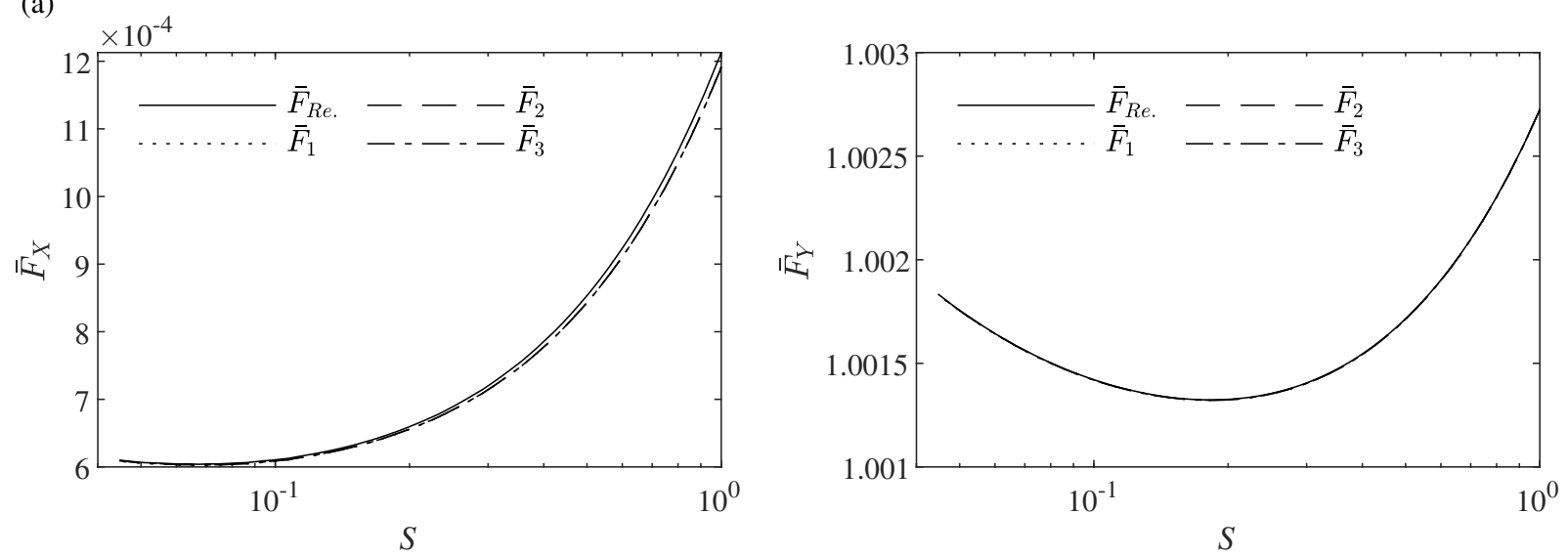

(b)
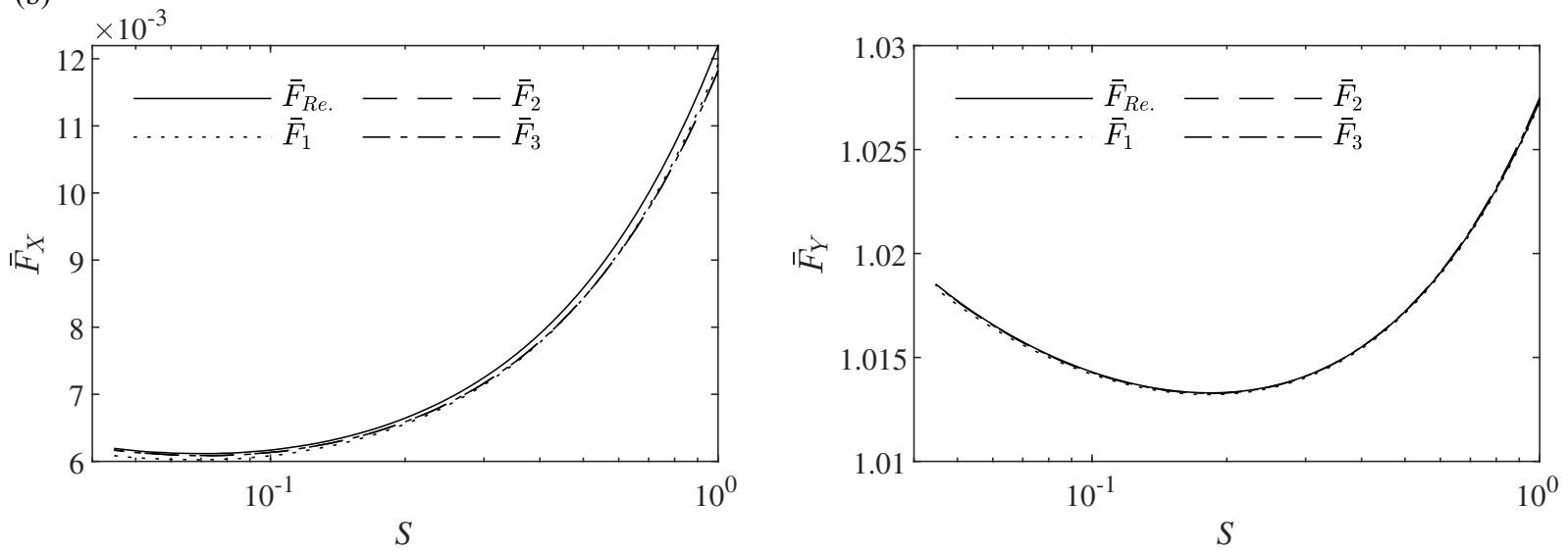

(c)
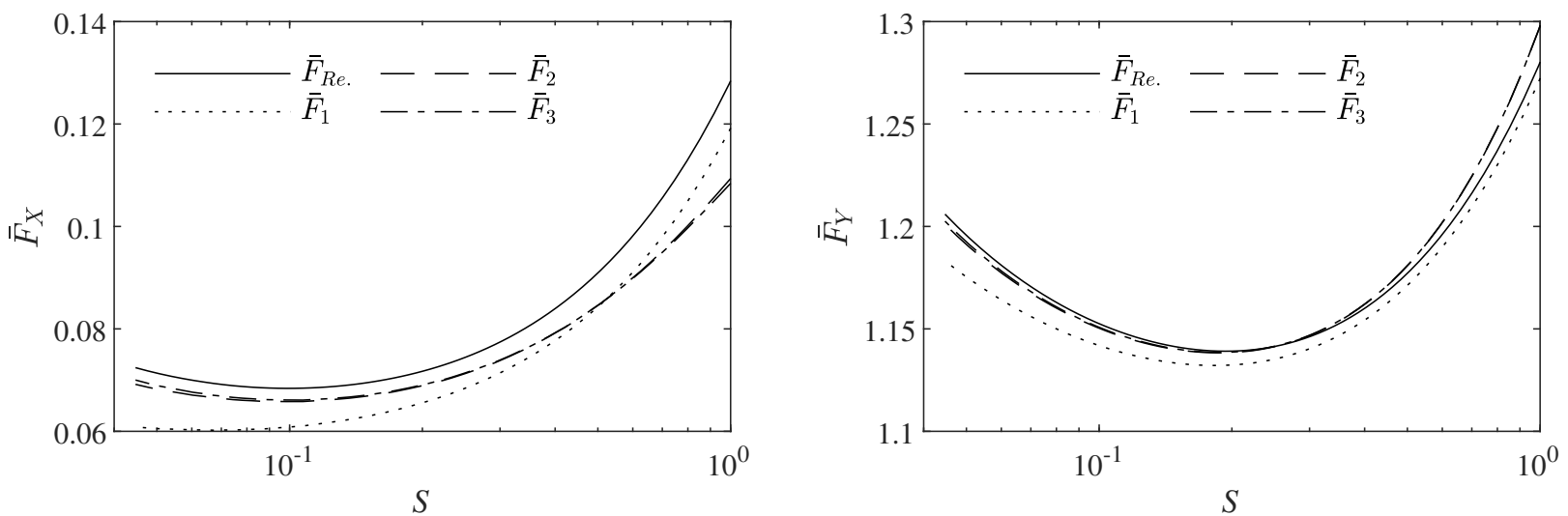

(d)
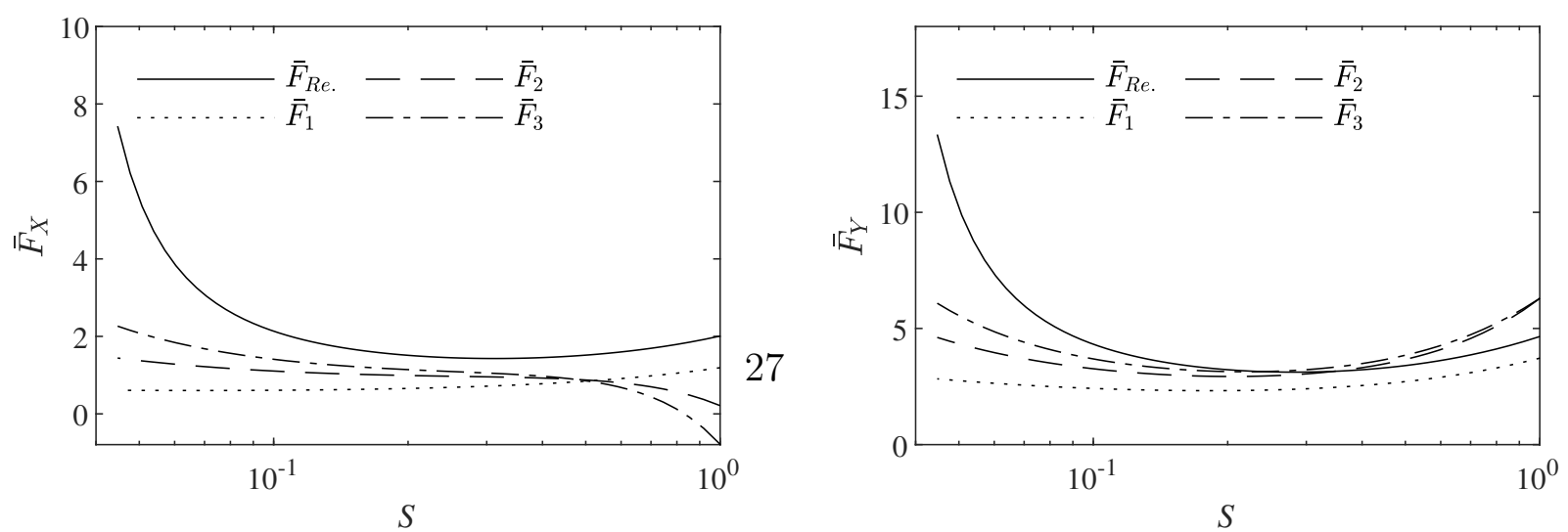

Figure 5: Semi-log plot for the dimensionless force components $\bar{F}_{X}$ and $\bar{F}_{Y}$ resulted from journal perturbation in $X$ and $Y$ directions for ungrooved journal bearing with $L / D=1$ (a) $\left(\delta X=0.0001, \delta X^{\prime}=0.0001, \delta Y=\right.$ 0.0001 and $\left.\delta Y^{\prime}=0.0001\right),(\mathrm{b})\left(\delta X=0.001, \delta X^{\prime}=0.001, \delta Y=0.001\right.$ and $\left.\delta Y^{\prime}=0.001\right),(\mathrm{c})(\delta X=$ $0.01, \delta X^{\prime}=0.01, \delta Y=0.01$ and $\left.\delta Y^{\prime}=0.01\right)$ and $(\mathrm{d})\left(\delta X=0.1, \delta X^{\prime}=0.1, \delta Y=0.1\right.$ and $\left.\delta Y^{\prime}=0.1\right)$. 


\subsection{Dynamic results}

\subsubsection{Stability Analysis}

In this section, the calculated bearing coefficients are used to investigate the dynamic analysis of the elastic rotor system presented in Figure 2. Initially, the stability of the autonomous dynamical system in Eq. 52 is investigated. This is done by evaluating the dimensionless threshold mass $\bar{M}_{t h}=m c \Omega_{t h}^{2} / W$ which implicitly includes the threshold speed of the system. The dimensionless threshold mass $\bar{M}_{t h}$ can be evaluated using the eigenvalues of the Jacobian matrix $\mathbf{J}(\mathbf{x}, \bar{M})$ in Eq. (54) at the fixed points. The fixed points can be obtained by solving the $\mathbf{f}(\mathbf{x}, \bar{M})=0$ in Eq. (53). This results in $\mathbf{x}^{*}=$ $\left[\begin{array}{llllllll}0 & 0 & 0 & 0 & 0 & 0 & 2 / K_{s} & 0\end{array}\right]^{T}$.

$$
\mathbf{J}(\mathbf{x}, \bar{M})=\frac{\partial \mathbf{x}^{\prime}}{\partial \mathbf{x}}=\left[\begin{array}{cccccccc}
0 & 1 & 0 & 0 & 0 & 0 & 0 & 0 \\
J_{21} & J_{22} & J_{23} & J_{24} & \frac{K_{s}}{2 M_{J}} & 0 & 0 & 0 \\
0 & 0 & 0 & 1 & 0 & 0 & 0 & 0 \\
J_{41} & J_{42} & J_{43} & J_{44} & 0 & 0 & \frac{K_{s}}{2 M_{J}} & 0 \\
0 & 0 & 0 & 0 & 0 & 1 & 0 & 0 \\
\frac{K_{s}}{M_{D}} & 0 & 0 & 0 & -\frac{K_{s}}{M_{D}} & 0 & 0 & 0 \\
0 & 0 & 0 & 0 & 0 & 0 & 0 & 1 \\
0 & 0 & \frac{K_{s}}{M_{D}} & 0 & 0 & 0 & -\frac{K_{s}}{M_{D}} & 0
\end{array}\right]
$$

where, 


$$
\begin{aligned}
J_{21}= & -\frac{K_{s}}{2 M_{J}}\left(K_{X X}+K_{X X X} x_{1}+K_{X X Y} x_{3}+C_{X X X} x_{2}+C_{X X Y} x_{4}+\frac{1}{2} K_{X X X X} x_{1}^{2}\right. \\
& \left.+K_{X X X Y} x_{1} x_{3}+\frac{1}{2} K_{X X Y Y} x_{3}^{2}+C_{X X X X} x_{1} x_{2}+C_{X X X Y} x_{1} x_{4}+C_{X X Y X} x_{3} x_{2}+C_{X X Y Y} x_{3} x_{4}\right) \\
J_{22}= & -\frac{1}{M_{J}}\left(C_{X X}+C_{X X X} x_{1}+C_{X Y X} x_{3}+\frac{1}{2} C_{X X X X} x_{1}^{2}+\frac{1}{2} C_{X Y Y X} x_{3}^{2}+C_{X X Y X} x_{1} x_{3}\right) \\
J_{23}= & -\frac{1}{M_{J}}\left(K_{X Y}+K_{X X Y} x_{1}+K_{X Y Y} x_{3}+C_{X Y X} x_{2}+C_{X Y Y} x_{4}+\frac{1}{2} K_{X Y Y Y} x_{3}^{2}\right. \\
& \left.+\frac{1}{2} K_{X X X Y} x_{1}^{2}+K_{X X Y Y} x_{1} x_{3}+C_{X Y Y X} x_{3} x_{2}+C_{X Y Y Y} x_{3} x_{4}+C_{X X Y X} x_{1} x_{2}+C_{X X Y Y} x_{1} x_{4}\right) \\
J_{24}= & -\frac{1}{M_{J}}\left(C_{X Y}+C_{X X Y} x_{1}+C_{X Y Y} x_{3}+\frac{1}{2} C_{X X X Y} x_{1}^{2}+\frac{1}{2} C_{X Y Y Y} x_{3}^{2}+C_{X X Y Y} x_{1} x_{3}\right) \\
J_{41}= & -\frac{1}{M_{J}}\left(K_{Y X}+K_{Y X X} x_{1}+K_{Y X Y} x_{3}+C_{Y X X} x_{2}+C_{Y X Y} x_{4}+\frac{1}{2} K_{Y X X X} x_{1}^{2}\right. \\
& \left.+K_{Y X X Y} x_{1} x_{3}+\frac{1}{2} K_{Y X Y Y} x_{3}^{2}+C_{Y X X X} x_{1} x_{2}+C_{Y X X Y} x_{1} x_{4}+C_{Y X Y X} x_{3} x_{2}+C_{Y X Y Y} x_{3} x_{4}\right) \\
J_{42}= & -\frac{1}{M_{J}}\left(C_{Y X}+C_{Y X X} x_{1}+C_{Y Y X} x_{3}+\frac{1}{2} C_{Y X X X} x_{1}^{2}+\frac{1}{2} C_{Y Y Y X} x_{3}^{2}+C_{Y X Y X} x_{1} x_{3}\right) \\
J_{43}= & -\frac{K_{s}}{2 M_{J}}-\frac{1}{M_{J}}\left(K_{Y Y}+K_{Y X Y} x_{1}+K_{Y Y Y} x_{3}+C_{Y Y X} x_{2}+C_{Y Y Y} x_{4}+\frac{1}{2} K_{Y Y Y Y} x_{3}^{2}\right. \\
& \left.+\frac{1}{2} K_{Y X X Y} x_{1}^{2}+K_{Y X Y Y} x_{1} x_{3}+C_{Y Y Y X} x_{3} x_{2}+C_{Y Y Y Y} x_{3} x_{4}+C_{Y X Y X} x_{1} x_{2}+C_{Y X Y Y} x_{1} x_{4}\right) \\
J_{44}= & -\frac{1}{M_{J}}\left(C_{Y Y}+C_{Y X Y} x_{1}+C_{Y Y Y} x_{3}+\frac{1}{2} C_{Y X X Y} x_{1}^{2}+\frac{1}{2} C_{Y Y Y Y} x_{3}^{2}+C_{Y X Y Y} x_{1} x_{3}\right) \\
& \\
&
\end{aligned}
$$


Evaluating the Jacobian matrix at fixed points $\mathbf{x}=\mathbf{x}^{*}$, results in

$$
\mathbf{J}(\overline{\mathbf{M}})_{\left(\mathbf{x}=\mathbf{x}^{*}\right)}=\left[\begin{array}{cccccccc}
0 & 1 & 0 & 0 & 0 & 0 & 0 & 0 \\
-\frac{K_{s}}{2 M_{J}}-\frac{K_{X X}}{M_{J}} & -\frac{C_{X X}}{M_{J}} & -\frac{K_{X Y}}{M_{J}} & -\frac{C_{X Y}}{M_{J}} & \frac{K_{s}}{2 M_{J}} & 0 & 0 & 0 \\
0 & 0 & 0 & 1 & 0 & 0 & 0 & 0 \\
-\frac{K_{Y X}}{M_{J}} & -\frac{C_{Y X}}{M_{J}} & -\frac{K_{s}}{2 M_{J}}-\frac{K_{Y Y}}{M_{J}} & -\frac{C_{Y Y}}{M_{J}} & 0 & 0 & \frac{K_{s}}{2 M_{J}} & 0 \\
0 & 0 & 0 & 0 & 0 & 1 & 0 & 0 \\
\frac{K_{s}}{M_{D}} & 0 & 0 & 0 & -\frac{K_{s}}{M_{D}} & 0 & 0 & 0 \\
0 & 0 & 0 & 0 & 0 & 0 & 0 & 1 \\
0 & 0 & \frac{K_{s}}{M_{D}} & 0 & 0 & 0 & -\frac{K_{s}}{M_{D}} & 0
\end{array}\right]
$$

Evaluating the Jacobian matrix eigenvalues $\left(\lambda_{1}, \lambda_{2}, \cdots, \lambda_{8}\right)$ then reorder them in descending order such as $\operatorname{Re}\left(\lambda_{1}\right) \geq \operatorname{Re}\left(\lambda_{2}\right) \geq \cdots \geq \operatorname{Re}\left(\lambda_{n}\right)$. The value of the $\bar{M}$ where $\operatorname{Re}\left(\lambda_{1}\right)=0$ is the threshold value $\bar{M}_{t h}$. This analysis is repeated for the available range of Sommerfeld number.

These results are presented on Figure 6-a, b. In Figure 6, the threshold mass is plotted versus the eccentricity ratio and in Figure 6+b, the threshold mass is plotted versus the dimensionless Sommerfeld number. The plot is done for several values of dimensionless shaft stiffness ranges $K_{s}=1$ to $K_{s}=10000$.

To investigate the stability of the periodic solution when the rotor speed exceeds the threshold speed the Monodromy matrix are evaluated from the Floquet multipliers. For the details of the Monodromy analysis, see for example(Nayfeh and Balachandran 1995, Seydel 2010, Miraskari et al. 2018b). If the eigenvalues of Monodromy matrix is less than one the periodic solution is stable limit cycle i.e. supercritical bifurcation and if the eigenvalue of the Monodromy matrix is greater than one the periodic solution is unstable i.e. subcritical bifurcation. This is plotted in Fig $[6$ using solid and dotted lines where the solid lines indicate 
supercritical bifurcation, and the dotted lines represent subcritical bifurcation solution. The results of the figure show that for the present dynamical model decreasing the stiffness of the rotor shaft increase the possibility of subcritical Hopf bifurcation. 
(a)

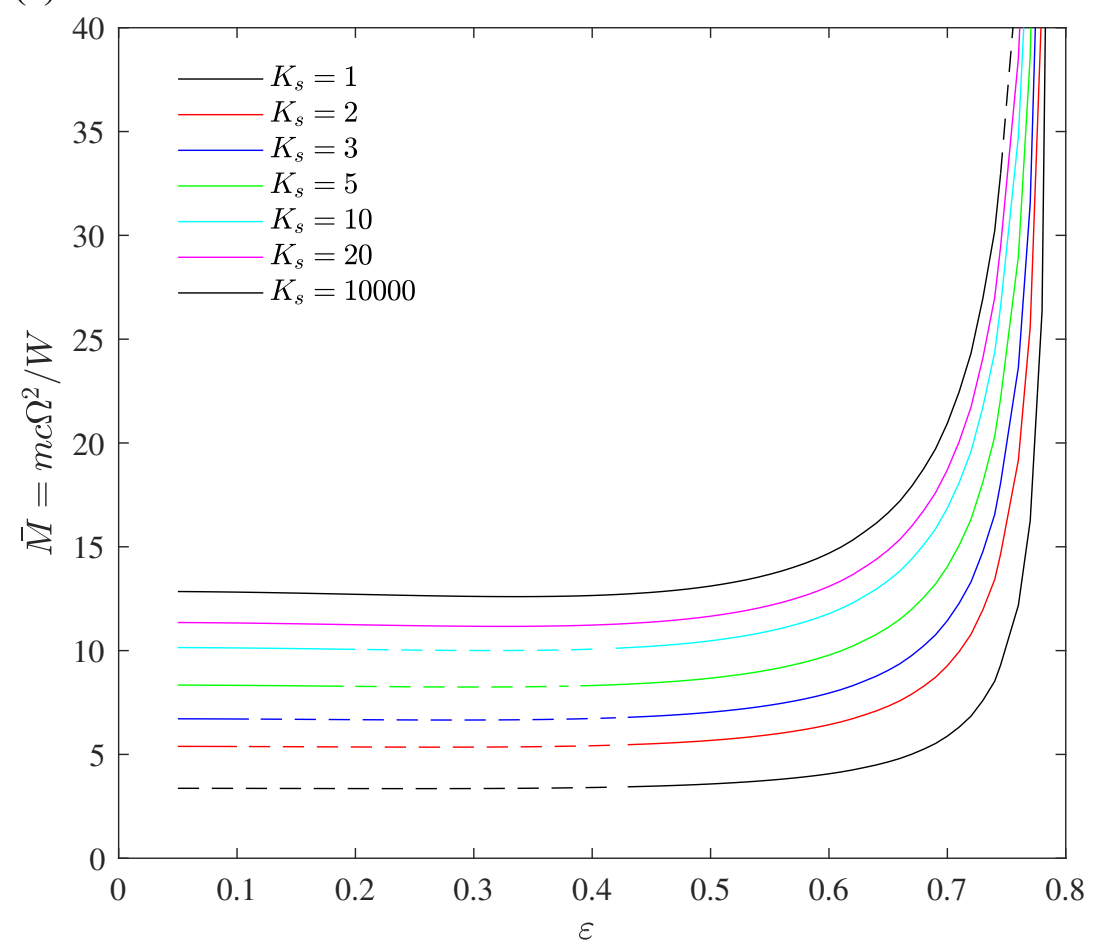

(b)

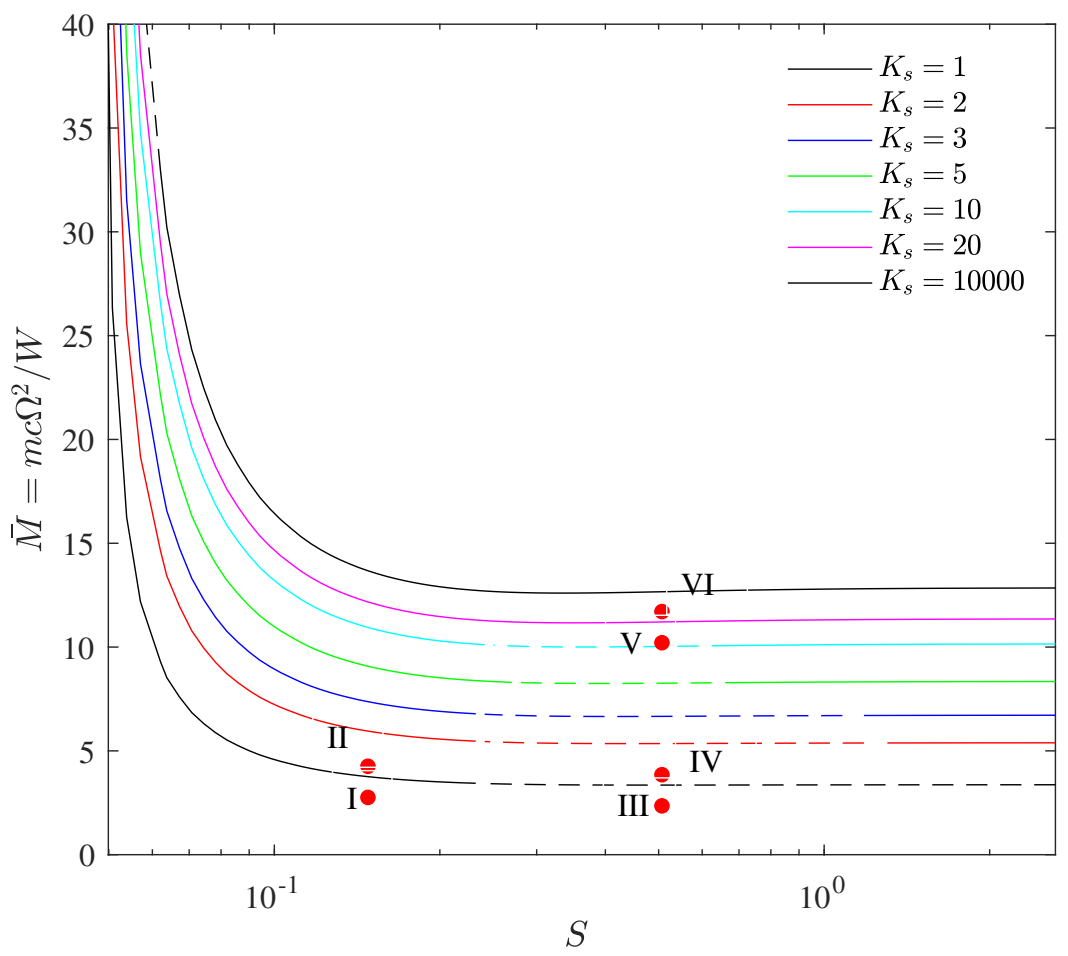

Figure 6: (Color online) Rotor stability curves: (a) Threshold mass versus the eccentricity ratio. (b)Threshold mass versus Sommerfeld No. . 


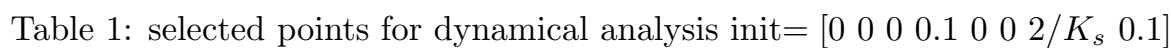

\begin{tabular}{llllll}
\hline Operating Point & $K_{s}$ & $\bar{M}$ & Sommerfeld number $S$ & Eccentricity ratio $\varepsilon$ & Bifurcation type \\
\hline I & 1 & $\bar{M}_{t h}-1$ & 0.148 & 0.55 & Supercritical \\
II & 1 & $\bar{M}_{t h}+0.5$ & 0.148 & 0.55 & Supercritical \\
III & 1 & $\bar{M}_{t h}-1$ & 0.507 & 0.24 & subcritical \\
IV & 1 & $\bar{M}_{t h}+0.5$ & 0.507 & 0.24 & subcritical \\
V & 20 & $\bar{M}_{t h}-1$ & 0.507 & 0.24 & Supercritical \\
VI & 20 & $\bar{M}_{t h}+0.5$ & 0.507 & 0.24 & Supercritical \\
\hline
\end{tabular}

\subsubsection{Time response comparative study}

In this section, selected points form the stability diagram I, II, III, IV, V and VI are investigated using orbit plots of the shaft geometrical center. The input data for these cases are shown in Table 1 and presented in Figure 6. The comparative analysis is done using four different analyses. The first analysis is based on the linear first order approximation of the bearing forces (LA). This analysis can be done using Eq. (52) while eliminating the second and the third order bearing coefficients. The second analysis is based on the nonlinear second order approximation of the bearing forces and this is done while reducing the third order coefficients to zero (NLA2). The third analysis is based on the nonlinear third order force approximation (NLA3) as stated in Eq.(52). The last analysis is based on the nonlinear force evaluated directly from Reynolds equation (RNL). In this analysis, the Reynolds equation is solved every time step to evaluate the bearing force.

The six selected operating points are listed in Table 1. All the cases are for $L / D=1$. The first two cases I, II are at $S=0.148$, the second two cases III, IV are at $S=0.507$ and the last two cases V, VI are at $S=0.507$ and $K_{s}=20$. The cases I, III and V are below the threshold speed and the cases II, IV and VI are above the threshold speed. 

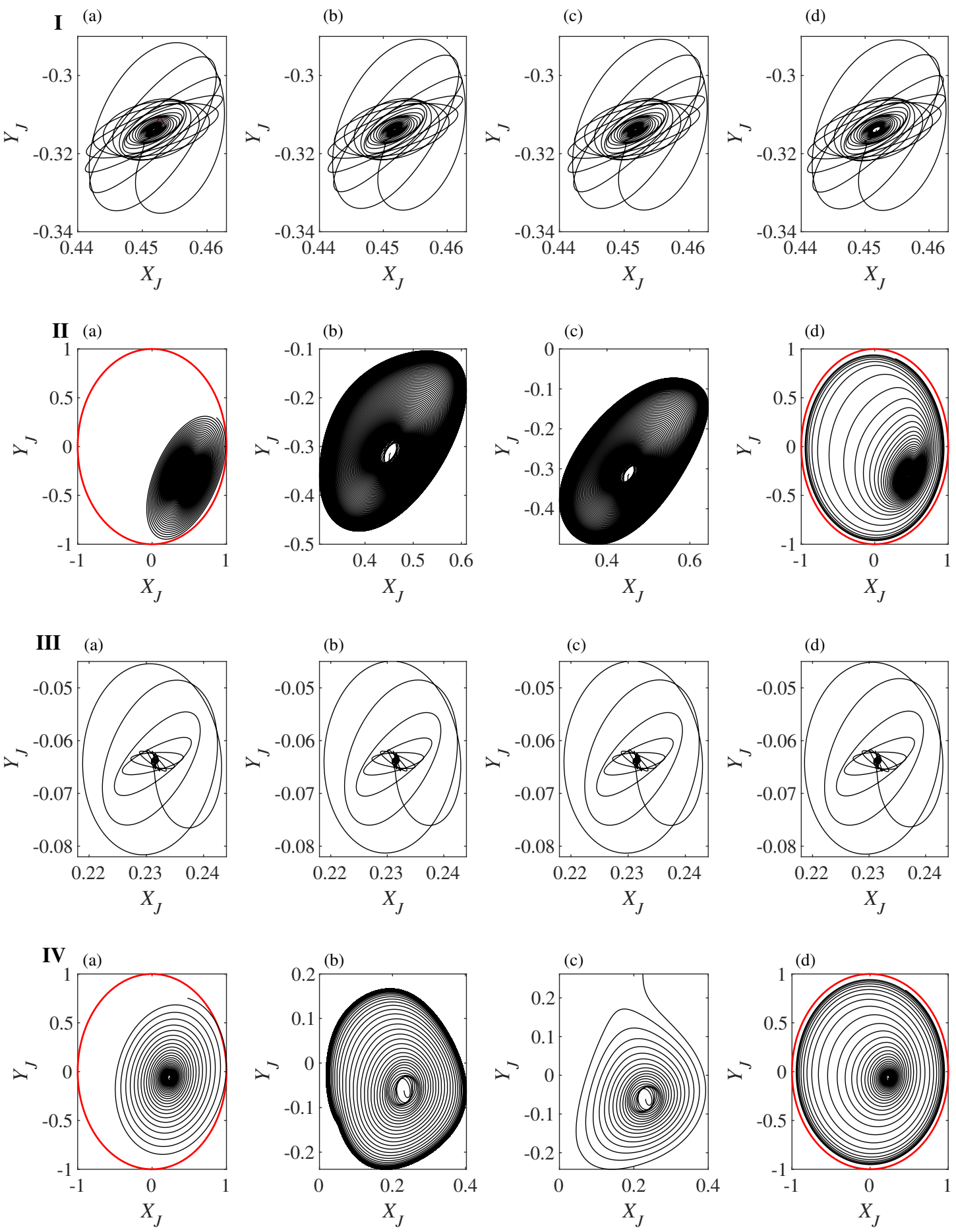

Figure 7: Orbit plot for the journal geometrical center at different operating points, point I, II, III and IV as shown in Table 1. For all cases (a) $1^{\text {st }}$ order (LA) (b) $2^{\text {nd }}$ order (NLA2) (c) $3^{\text {rd }}$ order(NLA3) (d) nonlinear (RNL). $K_{s}=1$, Init $=\left[\begin{array}{llllllll}0 & 0 & 0 & 0.1 & 0 & 0 & 2 / K_{s} & 0.1\end{array}\right]$ 

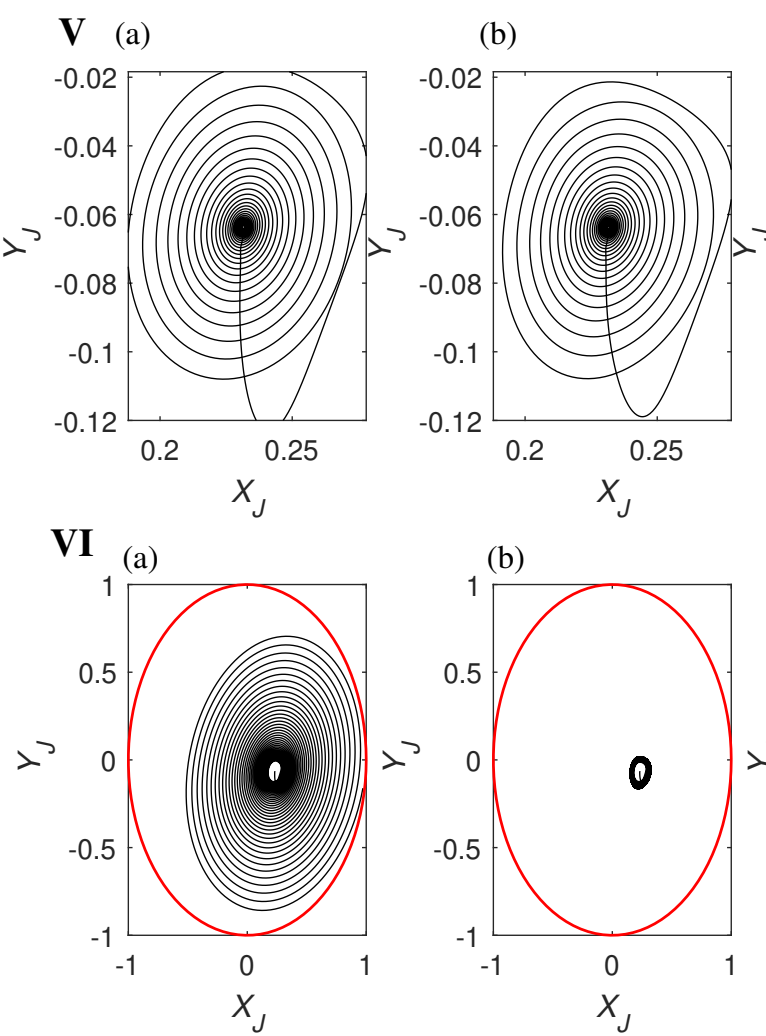

(b)

(c)

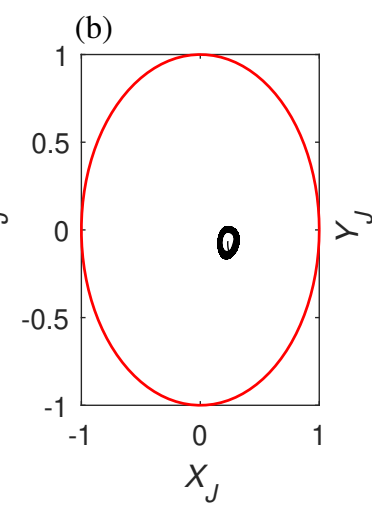

(d)
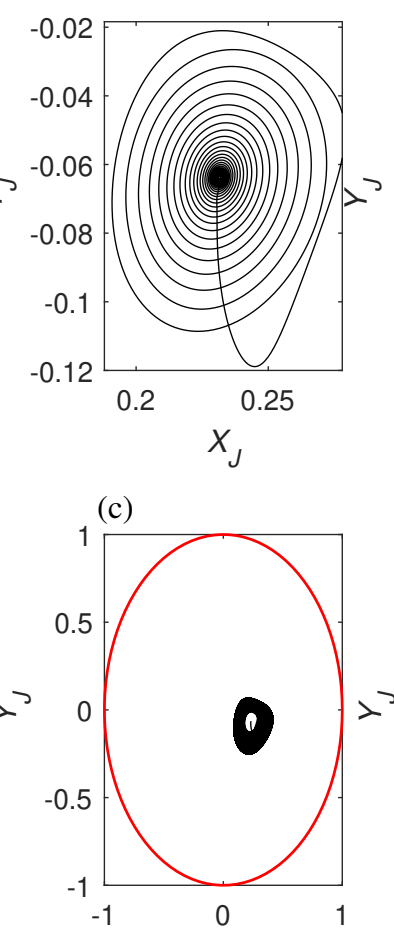
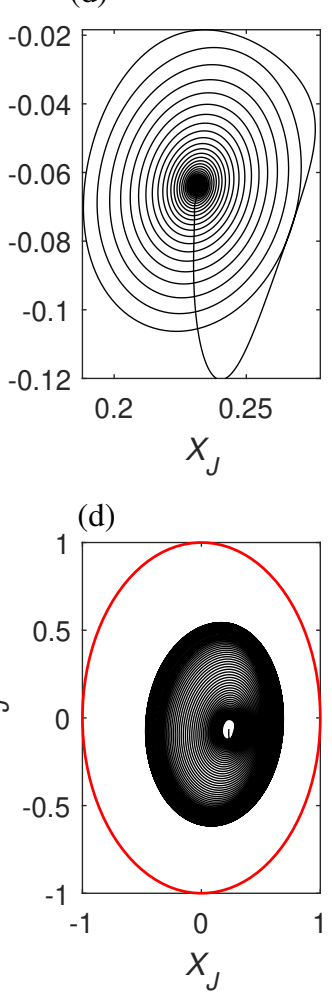

Figure 8: Orbit plot for the journal geometrical center two operating conditions V and VI as shown in Table 1. For all cases (a) $1^{\text {st }}$ order (LA) (b)2 $2^{\text {nd }}$ order (NLA2) (c) $3^{\text {rd }} \operatorname{order(NLA3)~(d)~nonlinear~(RNL).~}$

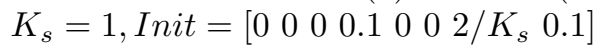

For case I and case III where both of them are below the threshold speed the four analyses give the same response as shown in the first and third row of Figure 7. Case II and case IV are above the threshold velocity for supercritical and subcritical cases according to the Monodromy bifurcation analysis. For case II, the response based on the linear analysis shows instability as the rotor hits the journal bearing. Both analyses based on the second order coefficients (NLA2) and third order coefficients (NLA3) show a stable limit cycle. However, the analysis based on direct solution of Reynolds (RNL) shows bifurcation leads to unstable limit cycle which then jumps to a larger stable limit cycle. This behavior for journal bearings is previously reported in (Chouchane and Amamou 2011, Sghir and Chouchane 2014).

For case IV which is subcritical according to the Monodromy analysis. The first order 
analysis shows unstable behavior above threshold speed which is basically expected because analysis based on first order (LA) is insensitive to bifurcation type(Miraskari et al. 2018b). In this case, the analyses based on NLA2 and NLA3 do not show the same response. The analysis based on second order coefficients shows a stable limit cycle while the analysis based on third order coefficients show unstable limit cycle. In this case, the nonlinear analysis RNL agrees with (NLA3) in showing unstable limit cycle. However, the RNL response shows a jump from unstable limit cycle to bigger stable limit cycle as shown Figure 7fIV-d. The explanation for the difference between the third order coefficient and RNL can be explained by the fact that the coefficients based on NLA3 is only valid in the neighborhood of the steady state point and as the rotor receding from this point, these coefficients become not accurate as shown in the perturbation analysis.

Another two cases, V and VI, using higher rotor dimensionless stiffness are investigated. The dimensionless stiffness in this condition is $K_{s}=20$. The stability diagram shows that all bifurcation for this case is supercritical. The orbit plot for the journal geometrical center is plotted at $S=0.507$. the first case $\mathrm{V}$ is below the threshold speed and the second case VI is above the threshold speed. The results show that for case V below the threshold speed all the four analysis show the same response. For case VI which is higher than the threshold speed the analysis based on first order coefficients show growing of the response until the journal hits the bearing. The analyses NLA2, NLA3 and RNL show stable limit cycle. The size of the limit cycle is not the same for the three cases. This can be explained by the fact that the bearing coefficients are evaluated to be correct in the vicinity of the steady state point.

\section{Conclusion}

In the present paper, the linear and nonlinear second and third order bearing coefficients are obtained using infinitesimal perturbation method. The obtained equations are 
solved using finite difference method. The effect of the mesh sensitivity on the obtained parameters are investigated. The validity range of bearing coefficients are investigated using perturbation analysis.

The obtained parameters are used to investigate the stability of a flexible rotor supported by two symmetric journal bearings. Eigenvalue analysis is used to evaluate the threshold speed. Monodromy analysis is used to obtain the bifurcation type above the threshold speed. The results of the orbit plot analysis show that below the threshold speed the analysis based on linear and nonlinear coefficients is compatible with that based on direct solution of Reynolds equation. Above the threshold speed the linear analysis is insensitive to limit cycles. The second and third bearing coefficient can predict the existence of limit cycles. The analysis based on third order coefficient giving better prediction than the second order. The analysis based on the bearing coefficients are closer to the analysis based on the complete solution of Reynolds equation at higher rotor dimensionless stiffness.

\section{Funding Information}

The authors declare that they do not receive any funds from any organization for this research.

\section{Conflict of interest}

The authors declare that they have no conflict of interest.

\section{References}

Akers, A., Michaelson, S., Cameron, A., . Stability contours for a whirling finite journal bearing. Journal of Lubrication Technology 93, 177-183. doi:https://doi.org/10.1115/1.3451510. 10.1115/1.3451510.

Arregui, I., Vázquez, C., 2001. Finite element solution of a reynolds-koiter coupled problem for the elastic journal-bearing. Computer methods in applied mechanics and engineering 190, 2051-2062. doi https: //doi.org/10.1016/S0045-7825(00)00221-8. 
Awasthi, R.K., Jain, S.C., Sharma, S.C., 2006. Finite element analysis of orifice-compensated multiple hole-entry worn hybrid journal bearing. Finite elements in analysis and design 42, 1291-1303. doi https : //doi.org/10.1016/j.finel.2006.06.007.

Bai, C., Zheng, D., Hure, R., Saleh, R., Carvajal, N., Morrison, G., 2019. The impact of journal bearing wear on an electric submersible pump in two-phase and three-phase flow. Journal of Tribology 141. doi:https://doi.org/10.1115/1.4042773

Castro, H.F., Cavalca, K.L., Nordmann, R., . Whirl and whip instabilities in rotor-bearing system considering a nonlinear force model. Journal of Sound and Vibration 317, 273-293. doi https: //doi.org/10.1016/j.jsv.2008.02.047

Chasalevris, A., 2020. Stability and hopf bifurcations in rotor-bearing-foundation systems of turbines and generators. Tribology International 145, 106154. doi:https://doi.org/10.1016/j.triboint.2019. 106154.

Chasalevris, A., Sfyris, D., . Evaluation of the finite journal bearing characteristics, using the exact analytical solution of the reynolds equation. Tribology International 57, 216-234. doi:https://doi.org/10.1016/ j.triboint.2012.08.011.

Chouchane, M., Amamou, A., 2011. Bifurcation of limit cycles in fluid film bearings. International Journal of Non-Linear Mechanics 46, 1258-1264. doi:https://doi.org/10.1016/j.ijnonlinmec.2011.06.005.

Chun, S.M., Khonsari, M.M., 2016. Wear simulation for the journal bearings operating under aligned shaft and steady load during start-up and coast-down conditions. Tribology International 97, 440-466. doi:https://doi.org/10.1016/j.triboint.2016.01.042.

Ciulli, E., Forte, P., Libraschi, M., Naldi, L., Nuti, M., 2018. Characterization of high-power turbomachinery tilting pad journal bearings: First results obtained on a novel test bench. Lubricants 6, 4. doi https: //doi.org/10.3390/lubricants6010004.

Dakel, M., Baguet, S., Dufour, R., 2014. Nonlinear dynamics of a support-excited flexible rotor with hydrodynamic journal bearings. Journal of Sound and Vibration 333, 2774-2799. doi:https://doi.org/ $10.1016 / j \cdot j s v .2013 .12 .021$.

Deepak, J.C., Noah, S.T., 1998. Experimental verification of subcritical whirl bifurcation of a rotor supported on a fluid film bearing. Journal of Tribology 120, 605-609. URL: https://doi.org/10.1115/1.2834593, doi:10.1115/1.2834593.

Ebrat, O., Mourelatos, Z.P., Vlahopoulos, N., Vaidyanathan, K., . Calculation of journal bearing dynamic characteristics including journal misalignment and bearing structural deformation(C). Tribology Transac- 
tions 47, 94-102. doi https://doi.org/10.1080/05698190490278994.

Ehrich, F.F., 1991. Some observations of chaotic vibration phenomena in high-speed rotordynamics. Journal of Vibration and Acoustics 113. doi https://doi.org/10.1115/1.2930154.

El-Sayed, T.A., Fatah, A.A.A., 2016. Performance of hydraulic turbocharger integrated with hydraulic energy management in swro desalination plants. Desalination 379, 85-92. doi:https://doi.org/10. $1016 / \mathrm{j} \cdot$ desal.2015.10.013.

Gao, G., Yin, Z., Jiang, D., Zhang, X., 2014. Numerical analysis of plain journal bearing under hydrodynamic lubrication by water. Tribology International 75, 31-38. doihttps://doi.org/10.1016/j.triboint. 2014.03 .009 .

Gao, G., Yin, Z., Jiang, D., Zhang, X., Wang, Y., 2016. Analysis on design parameters of water-lubricated journal bearings under hydrodynamic lubrication. Proceedings of the Institution of Mechanical Engineers, Part J: Journal of Engineering Tribology 230, 1019-1029. doi:https://doi.org/10.1177/ 1350650115623201 .

Gero, L.R., McC. Ettles, C.M., 1986. An evaluation of finite difference and finite element methods for the solution of the reynolds equation. ASLE transactions 29, 166-172. doi:https://doi.org/10.1080/ 05698198608981674

Goodwin, M.J., Ogrodnik, P.J., Roach, M.P., Fang, Y., 1997. Calculation and measurement of the stiffness and damping coefficients for a low impedance hydrodynamic bearing doi https://doi.org/10.1115/1. 2832480

Hassard, B.D., Hassard, B.D., Kazarinoff, N.D., Wan, Y.H., Wan, Y.W., 1981. Theory and applications of Hopf bifurcation. volume 41 of London Mathematical Society lecture note series. Cambridge University Press, Cambridge ; New York. URL: http://www.loc.gov/catdir/enhancements/fy0808/ 80049691-d.html.

Hili, M.A., Bouaziz, S., Maatar, M., Fakhfakh, T., Haddar, M., 2010. Hydrodynamic and elastohydrodynamic studies of a cylindrical journal bearing. Journal of hydrodynamics 22, 155-163. doi https: //doi.org/10.1016/S1001-6058(09)60041-X.

Huang, Y., Tian, Z., Chen, R., Cao, H., 2017. A simpler method to calculate instability threshold speed of hydrodynamic journal bearings. Mechanism and Machine Theory 108, 209-216. doi:https://doi.org/ $10.1016 / \mathrm{j} \cdot \mathrm{mechmachtheory} \cdot 2016.11 .009$.

Lund, J., Thomsen, K., 1978. A calculation method and data for the dynamic coefficients of oil-lubricated journal bearings. Topics in fluid film bearing and rotor bearing system design and optimization . 
Lund, J.W., 1965. The stability of an elastic rotor in journal bearings with flexible, damped supports. Journal of Applied Mechanics 32, 911-920. doi:https://doi.org/10.1115/1.3627335

Lund, J.W., 1974. Stability and damped critical speeds of a flexible rotor in fluid-film bearings. Journal of Engineering for Industry 96, 509-517. doi https://doi.org/10.1115/1.3438358

Mao, W., Han, X., Liu, G., Liu, J., 2016. Bearing dynamic parameters identification of a flexible rotorbearing system based on transfer matrix method. Inverse Problems in Science and Engineering 24, 372-392. doi:https://doi.org/10.1080/17415977.2015.1046860.

Miraskari, M., Hemmati, F., Gadala, M.S., 2018a. Nonlinear dynamics of flexible rotors supported on journal bearings - part i: Analytical bearing model. Journal of Tribology 140. doi:https://doi.org/10.1115/ 1.4037730

Miraskari, M., Hemmati, F., Gadala, M.S., 2018b. Nonlinear dynamics of flexible rotors supported on journal bearings - part ii: Numerical bearing model. Journal of Tribology 140, 021705. doi https: //doi.org/10.1115/1.4037731

Miura, T., Inoue, T., Kano, H., 2017. Nonlinear analysis of bifurcation phenomenon for a simple flexible rotor system supported by a full-circular journal bearing. Journal of Vibration and Acoustics 139. doi https: //doi.org/10.1115/1.4036098

Mori, T., 1968. Oil whip in the journal bearing. J. Jpn. Soc. Lubr. Eng. 13, 287-298.

Mourelatos, Z.P., 1985. Finite element analysis of elastohydrodynamic stern tube bearings (lubrication, journal, optimization). Ph.d. URL: http://search.proquest.com/docview/303380720?accountid= 37552. copyright - Copyright UMI - Dissertations Publishing 1985 Last updated - 2014-04-07 First page - n/a.

Muszynska, A., 1988. Stability of whirl and whip in rotor/bearing systems. Journal of Sound and Vibration 127, 49-64. doi/https://doi.org/10.1016/0022-460X (88)90349-5

Nair, V.P.S., Nair, K.P., 2004. Finite element analysis of elastohydrodynamic circular journal bearing with micropolar lubricants. Finite Elements in Analysis and Design 41, 75-89. doi/https://doi.org/10. 1016/j.finel.2004.04.001.

Nayfeh, A.H., Balachandran, B., 1995. Applied nonlinear dynamics: analytical, computational, and experimental methods. Wiley series in nonlinear science, Wiley, New York. URL: http://www.loc.gov/ catdir/bios/wiley041/94003659.html.

Nicoletti, R., 2013. Comparison between a meshless method and the finite difference method for solving the reynolds equation in finite bearings. Journal of Tribology 135. doi https://doi.org/10.1115/1. 
4024752

Nishimura, A., Inoue, T., Watanabe, Y., 2018. Nonlinear analysis and characteristic variation of self-excited vibration in the vertical rotor system due to the flexible support of the journal bearing. Journal of Vibration and Acoustics 140. doi:https://doi.org/10.1115/1.4037520

Novotný, P., Škara, P., Hliník, J., 2018. The effective computational model of the hydrodynamics journal floating ring bearing for simulations of long transient regimes of turbocharger rotor dynamics. International Journal of Mechanical Sciences 148, 611-619. doi:https://doi.org/10.1016/j.ijmecsci.2018. 09.025

On, S.Y., Kim, Y.S., You, J.I., Lim, J.W., Kim, S.S., 2018. Dynamic characteristics of composite tilting pad journal bearing for turbine/generator applications. J Composite Structures 201, 747-759. doi https: //doi.org/10.1016/j.compstruct.2018.06.095.

Qiu, Z., Tieu, A., 1996. The effect of perturbation amplitudes on eight force coefficients of journal bearings. Tribology transactions 39, 469-475. doi:https://doi.org/10.1080/10402009608983554.

Qiu, Z.L., Tieu, A.K., 1995. Misalignment effect on the static and dynamic characteristics of hydrodynamic journal bearings doi:https://doi.org/10.1115/1.2831542.

Salamone, D.J., 1984. Journal bearing design types and their applications to turbomachinery, in: PROCEEDINGS OF THE THIRTEENTH TURBOMACHINERY SYMPOSIUM, Texas A\&M University. Turbomachinery Laboratories.

Santos, N.D.S.A., Roso, V.R., Faria, M.T.C., . Review of engine journal bearing tribology in start-stop applications. Engineering Failure Analysis 108, 104344. doi https://doi.org/10.1016/j.engfailanal. 2019.104344

Schweizer, B., . Dynamics and stability of turbocharger rotors. Archive of Applied Mechanics 80, $1017-1043$. doi:https://doi.org/10.1007/s00419-009-0331-0

Seydel, R., 2010. Practical bifurcation and stability analysis. volume 5 of Interdisciplinary applied mathematics. Springer Science \& Business Media, New York.

Sghir, R., Chouchane, M., 2014. Prediction of the nonlinear hysteresis loop for fluid-film bearings by numerical continuation. Proceedings of the Institution of Mechanical Engineers, Part C: Journal of Mechanical Engineering Science 229, 651-662. URL: https://doi.org/10.1177/0954406214538618 doi: $10.1177 / 0954406214538618$

Shin, D., Palazzolo, A.B., 2020. Nonlinear analysis of a geared rotor system supported by fluid film journal bearings. Journal of Sound and Vibration 475, 115269. 
Smolík, L., Haj žman, M., Byrtus, M., 2017. Investigation of bearing clearance effects in dynamics of turbochargers. International Journal of Mechanical Sciences 127, 62-72. doi:https://doi.org/10.1016/ j.ijmecsci.2016.07.013.

Smolík, L., Rendl, J., Dyk, u., Polach, P., Hajžman, M., 2019. Threshold stability curves for a nonlinear rotor-bearing system. Journal of Sound and Vibration 442, 698-713. doi https://doi.org/10.1016/j . jsv.2018.10.042.

Tiwari, R., Lees, A.W., Friswell, M.I., 2004. Identification of dynamic bearing parameters: a review. Shock and Vibration Digest 36, 99-124.

Vance, J.M., Murphy, B., Zeidan, F., 2010. Machinery vibration and rotordynamics. John Wiley \& Sons, Hoboken, N.J.

Wada, S., Hayashi, H., Migita, M., 1971. Application of finite element method to hydrodynamic lubrication problems (part 1). Trans. JSME 37, 583-592. doi:https://doi.org/10.1299/kikai1938.37.593.

Wang, J.K., Khonsari, M.M., 2006. A new derivation for journal bearing stiffness and damping coefficients in polar coordinates. Journal of sound and vibration 290, 500-507. doi https://doi.org/10.1016/j. jsv.2005.04.019.

Weimin, W., Lihua, Y., Tiejun, W., Lie, Y., 2012. Nonlinear dynamic coefficients prediction of journal bearings using partial derivative method. Proceedings of the Institution of Mechanical Engineers, Part J: Journal of Engineering Tribology 226, 328-339. doi https://doi.org/10.1177/1350650111431526.

Yu, L., Qi, S., Geng, H., 2005. A generalized solution of elasto-aerodynamic lubrication for aerodynamic compliant foil bearings. Science in China Ser. E Engineering \& Materials Science 48, 414-429. doi https: //doi.org/10.1360/03ye0551.

Zhang, X., Yin, Z., Gao, G., Li, Z., 2015. Determination of stiffness coefficients of hydrodynamic waterlubricated plain journal bearings. Tribology International 85, 37-47. doi https://doi.org/10.1016/j . triboint.2014.12.019.

Zhang, X., Yin, Z., Jiang, D., Gao, G., Wang, Y., Wang, X., 2016. Load carrying capacity of misaligned hydrodynamic water-lubricated plain journal bearings with rigid bush materials. Tribology International 99, 1-13. doi:https://doi.org/10.1016/j.triboint.2016.02.038. 
Appendix A. Derivative of dimensionless oil-film thickness

In this appendix, a list of important formulae for evaluating the oil-film thickness derivation which are required for first, second and third order coefficient evaluation 


$$
\begin{aligned}
& \frac{\partial H_{0}}{\partial \phi}=-\varepsilon_{0} \sin \varphi_{0} \\
& H_{X}=\frac{\partial H}{\partial X}=\sin \phi \\
& H_{Y}=\frac{\partial H}{\partial Y}=\cos \phi \\
& H_{\phi X}=\frac{\partial^{2} H}{\partial \phi \partial X}=\cos \phi \\
& H_{\phi Y}=\frac{\partial^{2} H}{\partial \phi \partial Y}=-\sin \phi \\
& H_{X X}=\frac{\partial^{2} H}{\partial X^{2}}=\frac{1}{\varepsilon_{0}}\left(2 \sin \theta_{0} \cos \theta_{0} \sin \varphi_{0}-\cos ^{2} \theta_{0} \cos \varphi_{0}\right) \\
& H_{Y Y}=\frac{\partial^{2} H}{\partial Y^{2}}=\frac{1}{\varepsilon_{0}}\left(-2 \sin \theta_{0} \cos \theta_{0} \sin \varphi_{0}-\sin ^{2} \theta_{0} \cos \varphi_{0}\right) \\
& H_{X Y}=\frac{\partial^{2} H}{\partial X \partial Y}=\frac{1}{\varepsilon_{0}}\left(\sin \theta_{0} \cos \theta_{0} \cos \varphi_{0}+\cos ^{2} \theta_{0} \sin \varphi_{0}-\sin ^{2} \theta_{0} \sin \varphi_{0}\right) \\
& H_{\phi X X}=\frac{\partial^{3} H}{\partial \phi \partial X^{2}}=\frac{1}{\varepsilon_{0}}\left(2 \sin \theta_{0} \cos \theta_{0} \cos \varphi_{0}+\cos ^{2} \theta_{0} \sin \varphi_{0}\right) \\
& H_{\phi Y Y}=\frac{\partial^{3} H}{\partial \phi \partial Y^{2}}=\frac{1}{\varepsilon_{0}}\left(-2 \sin \theta_{0} \cos \theta_{0} \cos \varphi_{0}+\sin ^{2} \theta_{0} \sin \varphi_{0}\right) \\
& H_{\phi X Y}=\frac{\partial^{3} H}{\partial \phi \partial X \partial Y}=\frac{1}{\varepsilon_{0}}\left(-\sin \theta_{0} \cos \theta_{0} \sin \varphi_{0}+\cos ^{2} \theta_{0} \cos \varphi_{0}-\sin ^{2} \theta_{0} \cos \varphi_{0}\right) \\
& H_{X X X}=\frac{\partial^{3} H}{\partial X^{3}}=\frac{1}{\varepsilon_{0}^{2}}\left(-3 \sin \theta_{0} \cos ^{2} \theta_{0} \cos \varphi_{0}-\cos ^{3} \theta_{0} \sin \varphi_{0}\right) \\
& H_{Y Y Y}=\frac{\partial^{3} H}{\partial X^{3}}=\frac{1}{\varepsilon_{0}^{2}}\left(\sin ^{3} \theta_{0} \sin \varphi_{0}-3 \sin ^{2} \theta_{0} \cos \theta_{0} \cos \varphi_{0}\right) \\
& H_{X Y Y}=\frac{\partial^{3} H}{\partial X \partial Y^{2}}=\frac{1}{\varepsilon_{0}^{2}}\left(2 \cos ^{2} \theta_{0} \sin \theta_{0} \cos \varphi_{0}-\sin ^{3} \theta_{0} \cos \varphi_{0}-\sin ^{2} \theta_{0} \cos \theta_{0} \sin \varphi_{0}\right) \\
& H_{X X Y}=\frac{\partial^{3} H}{\partial Y \partial X^{2}}=\frac{1}{\varepsilon_{0}^{2}}\left(2 \sin ^{2} \theta_{0} \cos \theta_{0} \cos \varphi_{0}-\cos ^{3} \theta_{0} \cos \varphi_{0}+\cos ^{2} \theta_{0} \sin \theta_{0} \sin \varphi_{0}\right) \\
& H_{\phi X X X}=\frac{\partial^{4} H}{\partial \phi \partial X^{3}}=\frac{1}{\varepsilon_{0}^{2}}\left(3 \sin \theta_{0} \cos ^{2} \theta_{0} \sin \varphi_{0}-\cos ^{3} \theta_{0} \cos \varphi_{0}\right) \\
& H_{\phi Y Y Y}=\frac{\partial^{4} H}{\partial \phi \partial Y^{3}}=\frac{1}{\varepsilon_{0}^{2}}\left(\sin ^{3} \theta_{0} \cos \varphi_{0}+3 \sin ^{2} \theta_{0} \cos \theta_{0} \sin \varphi_{0}\right) \\
& H_{\phi X Y Y}=\frac{\partial^{4} H}{\partial \phi \partial X \partial Y^{2}}=\frac{1}{\varepsilon_{0}^{2}}\left(-2 \cos ^{2} \theta_{0} \sin \theta_{0} \sin \varphi_{0}+\sin ^{3} \theta_{0} \sin \varphi_{0}-\sin ^{2} \theta_{0} \cos \theta_{0} \cos \varphi_{0}\right) \\
& H_{\phi X X Y}=\frac{\partial^{4} H}{\partial \phi \partial Y \partial X^{2}}=\frac{1}{\varepsilon_{0}^{2}}\left(-2 \sin ^{2} \theta_{0} \cos \theta_{0} \sin \varphi_{0}+\cos ^{3} \theta_{0} \sin \varphi_{0}+\cos ^{2} \theta_{0} \sin \theta_{0} \cos \varphi_{0}\right)
\end{aligned}
$$




\section{Appendix B. Bearing first, second and third order coefficients}

Table B.1: List of the first and second order bearing coefficients for fully circular journal bearing at $L / D=1$

\begin{tabular}{|c|c|c|c|c|c|c|c|c|c|c|c|c|c|c|c|c|c|c|c|c|c|c|c|}
\hline$\varepsilon$ & $\theta_{0}$ & $S$ & $K_{X X}$ & $K_{X Y}$ & $K_{Y X}$ & $K_{Y Y}$ & $C_{X X}$ & $\begin{array}{l}C_{X Y}= \\
C_{Y X}\end{array}$ & $C_{Y Y}$ & $K_{X X X}$ & $K_{X X Y}$ & $K_{X Y Y}$ & $K_{Y X X}$ & $K_{Y X Y}$ & $K_{Y Y Y}$ & $C_{X X X}$ & $C_{X X Y}$ & $C_{X Y Y}$ & $C_{Y X X}$ & $C_{Y X Y}$ & $C_{Y Y Y}$ & $C_{X Y X}$ & $C_{Y Y X}$ \\
\hline 0.05 & 86.86 & 2.63 & 2.14 & -19.25 & 20.11 & 1.10 & 38.64 & 2.12 & 40.18 & 86.06 & -384.15 & -94.13 & 3.77 & 43.50 & -399.61 & 8.44 & 86.02 & -767.36 & -44.06 & 803.24 & 88.15 & 72.81 & 804.28 \\
\hline 0.10 & 83.78 & 1.30 & 2.12 & -9.54 & 10.22 & 1.13 & 19.33 & 2.10 & 20.35 & 43.43 & -93.69 & -47.01 & 3.83 & 22.33 & -99.36 & 6.89 & 43.48 & -187.87 & -20.99 & 203.34 & 44.90 & 36.83 & 204.20 \\
\hline 0.15 & 80.70 & 0.85 & 2.12 & -6.24 & 7.00 & 1.16 & 12.85 & 2.10 & 13.85 & 29.55 & -39.58 & -31.40 & 3.96 & 15.49 & -43.65 & 6.40 & 29.64 & -80.16 & -13.28 & 92.38 & 30.95 & 25.16 & 93.19 \\
\hline 0.20 & 77.62 & 0.62 & 2.11 & -4.54 & 5.44 & 1.21 & 9.56 & 2.10 & 10.70 & 22.79 & -20.42 & -23.61 & 4.14 & 12.30 & -24.04 & 6.12 & 22.90 & -42.18 & -9.29 & 53.70 & 24.39 & 19.50 & 54.48 \\
\hline 0.25 & 74.52 & 0.48 & 2.09 & -3.49 & 4.56 & 1.28 & 7.58 & 2.10 & 8.88 & 18.91 & -11.44 & -18.94 & 4.38 & 10.59 & -14.84 & 6.00 & 19.05 & -24.45 & -6.76 & 35.97 & 20.80 & 16.28 & 36.75 \\
\hline 0.30 & 71.40 & 0.39 & 2.08 & -2.75 & 4.01 & 1.37 & 6.23 & 2.10 & 7.74 & 16.44 & -6.37 & -15.80 & 4.69 & 9.69 & -9.67 & 5.88 & 16.61 & -14.57 & -4.96 & 26.54 & 18.80 & 14.25 & 27.32 \\
\hline 0.35 & 68.27 & 0.32 & 2.06 & -2.21 & 3.66 & 1.47 & 5.26 & 2.10 & 6.99 & 14.82 & -3.16 & -13.54 & 5.07 & 9.28 & -6.35 & 5.83 & 15.02 & -8.41 & -3.55 & 21.07 & 17.77 & 12.95 & 21.86 \\
\hline 0.40 & 65.11 & 0.26 & 2.05 & -1.77 & 3.43 & 1.60 & 4.51 & 2.09 & 6.48 & 13.72 & -0.88 & -11.79 & 5.54 & 9.27 & -3.93 & 5.78 & 13.97 & -4.14 & -2.38 & 17.77 & 17.46 & 12.10 & 18.59 \\
\hline 0.45 & 61.91 & 0.22 & 2.03 & -1.41 & 3.28 & 1.76 & 3.92 & 2.09 & 6.15 & 12.99 & 0.90 & -10.36 & 6.12 & 9.60 & -1.93 & 5.75 & 13.28 & -0.92 & -1.36 & 15.81 & 17.77 & 11.57 & 16.64 \\
\hline 0.50 & 58.66 & 0.18 & 2.01 & -1.10 & 3.20 & 1.96 & 3.43 & 2.09 & 5.96 & 12.54 & 2.42 & -9.11 & 6.84 & 10.29 & -0.02 & 5.73 & 12.89 & 1.74 & -0.43 & 14.74 & 18.69 & 11.29 & 15.61 \\
\hline 0.55 & 55.36 & 0.15 & 1.99 & -0.82 & 3.18 & 2.21 & 3.02 & 2.09 & 5.86 & 12.31 & 3.87 & -7.94 & 7.73 & 11.41 & 2.06 & 5.71 & 12.73 & 4.13 & 0.46 & 14.36 & 20.31 & 11.21 & 15.28 \\
\hline 0.60 & 51.97 & 0.12 & 1.97 & -0.56 & 3.20 & 2.51 & 2.67 & 2.09 & 5.86 & 12.31 & 5.33 & -6.77 & 8.89 & 13.08 & 4.60 & 5.74 & 12.82 & 6.50 & 1.36 & 14.62 & 22.84 & 11.36 & 15.59 \\
\hline 0.65 & 48.47 & 0.10 & 1.95 & -0.32 & 3.28 & 31 & 2.36 & 2.0 & 96 & 12.52 & 6.99 & -5.43 & 10.39 & 15.56 & 8.10 & 5.77 & 13.12 & 9.05 & 2.29 & 15.50 & 26.62 & 11.70 & 16.54 \\
\hline 0.70 & 44.82 & 0.08 & 1.93 & -0.08 & 3.41 & 3.44 & 2.08 & 2.09 & 6.16 & 12.99 & 9.01 & -3.76 & 12.44 & 19.31 & 13.37 & 5.85 & 13.72 & 12.08 & 3.33 & 17.17 & 32.37 & 12.31 & 18.32 \\
\hline 0.75 & 40.96 & 0.06 & 1.92 & 0.18 & 3.62 & 4.17 & 1.83 & 2.10 & 6.49 & 13.81 & 11.69 & -1.35 & 15.38 & 25.24 & 22.14 & 5.96 & 14.68 & 16.01 & 4.55 & 19.95 & 41.45 & 13.26 & 21.25 \\
\hline 0.80 & 36.78 & 0.04 & 1.91 & 0.48 & 3.93 & 5.27 & 1.58 & 2.11 & 7.00 & 15.12 & 15.63 & 2.67 & 19.86 & 35.40 & 38.64 & 6.12 & 16.16 & 21.64 & 6.07 & 24.53 & 56.84 & 14.69 & 26.06 \\
\hline 0.85 & 32.14 & 0.03 & 1.91 & 0.8 & 4.4 & & 1.34 & 2.14 & & 17.36 & 22.16 & 10.50 & 7.61 & & 75.02 & 6.41 & 18.68 & 30.96 & 8.29 & 32.82 & 86.54 & 17.11 & 34.71 \\
\hline 0.90 & 26.68 & 0.02 & 1.94 & 1.39 & 5.34 & 10.66 & 1.10 & 2.18 & 9.29 & 21.55 & 35.58 & 30.91 & 43.67 & 103.47 & 182.38 & 6.83 & 23.18 & 49.51 & 11.88 & 50.09 & 157.65 & 21.42 & 52.71 \\
\hline 0.95 & 19.51 & 0.01 & 2.01 & 2.54 & 7.53 & 21.28 & 0.81 & 2.28 & 12.73 & 32.31 & 77.77 & 121.72 & 94.91 & 303.28 & 777.02 & 7.75 & 34.54 & 107.07 & 20.40 & 105.03 & 444.04 & 32.28 & 109.82 \\
\hline
\end{tabular}


Table B.2: List of the third order bearing coefficients for fully circular journal bearing at $L / D=1$

\begin{tabular}{|c|c|c|c|c|c|c|c|c|c|c|c|c|c|c|c|c|c|c|c|c|c|c|}
\hline$\varepsilon$ & $\theta_{0}$ & $S$ & $K_{X X X X}$ & $K_{X Y Y Y}$ & $K_{X X X Y}$ & $K_{X X Y Y}$ & $C_{X X X X}$ & $C_{X X X Y}$ & $C_{X Y Y X}$ & $C_{X Y Y Y}$ & $C_{X X Y X}$ & $C_{X X Y Y}$ & $K_{Y X X X}$ & $K_{Y Y Y Y}$ & $K_{Y X X Y}$ & $K_{Y X Y Y}$ & $C_{Y X X X}$ & $C_{Y X X Y}$ & $C_{Y Y Y X}$ & $C_{Y Y Y Y}$ & $C_{Y X Y X}$ & $C_{Y X Y Y}$ \\
\hline 0.05 & 86.86 & 2.63 & 41.59 & 7484.01 & 51.34 & -2937.50 & -91.91 & 3442.44 & -15261.74 & -4608.90 & 3178.17 & -15214.64 & -112.80 & -2603.33 & 1742.78 & -7935.82 & -11.80 & 150.19 & 1757.04 & -15911.93 & 201.47 & 1753.75 \\
\hline 0.10 & 83.78 & 1.30 & 38.22 & 821.02 & 41.85 & -742.79 & -39.76 & 869.38 & -1821.28 & -1144.12 & 802.75 & -1797.50 & -52.38 & -655.42 & 442.04 & -964.14 & -10.07 & 76.02 & 463.23 & -1948.45 & 100.14 & 460.13 \\
\hline 0.15 & 80.70 & 0.85 & 37.75 & 180.42 & 34.26 & -336.31 & -22.65 & 395.53 & -491.13 & -504.72 & 365.54 & -474.95 & -31.76 & -295.85 & 203.31 & -270.47 & -9.15 & 52.01 & 223.50 & -553.20 & 68.01 & 220.42 \\
\hline 0.20 & 77.62 & 0.62 & 38.09 & 34.73 & 30.91 & -193.57 & -13.62 & 230.38 & -174.74 & -280.11 & 213.22 & -162.26 & -20.55 & -170.31 & 120.82 & -103.73 & -8.29 & 40.58 & 140.62 & -215.25 & 52.72 & 137.48 \\
\hline 0.25 & 74.52 & 0.48 & 39.12 & -12.41 & 29.59 & -127.10 & -7.48 & 154.91 & -65.51 & -175.42 & 143.68 & -55.14 & -12.88 & -112.27 & 83.74 & -44.87 & -7.36 & 34.35 & 103.45 & -94.50 & 44.34 & 100.19 \\
\hline 0.30 & 71.40 & 0.39 & 40.52 & -31.64 & 30.03 & -90.32 & -2.94 & 114.68 & -17.93 & -117.19 & 106.66 & -8.89 & -6.77 & -80.77 & 64.93 & -18.43 & -6.28 & 30.86 & 84.82 & -39.45 & 39.49 & 81.41 \\
\hline 0.35 & 68.27 & 0.32 & 42.51 & -40.75 & 31.53 & -67.47 & 0.95 & 91.54 & 6.40 & -80.74 & 85.45 & 14.61 & -1.26 & -61.61 & 55.18 & -4.02 & -4.99 & 29.20 & 75.45 & -8.99 & 36.98 & 71.83 \\
\hline 0.40 & 65.11 & 0.26 & 45.00 & -46.08 & 34.25 & -51.62 & 4.34 & 77.63 & 21.23 & -55.13 & 72.77 & 28.91 & 4.17 & -48.78 & 50.87 & 5.75 & -3.45 & 28.94 & 71.76 & 11.62 & 36.16 & 67.86 \\
\hline 0.45 & 61.91 & 0.22 & 48.17 & -49.89 & 38.21 & -39.43 & 7.52 & 69.44 & 32.04 & -35.08 & 65.41 & 39.43 & 9.97 & -39.16 & 50.51 & 14.13 & -1.59 & 29.98 & 72.29 & 28.87 & 36.88 & 68.03 \\
\hline 0.50 & 58.66 & 0.18 & 52.18 & -53.17 & 43.69 & -28.86 & 10.64 & 65.18 & 41.62 & -17.33 & 61.72 & 48.92 & 16.61 & -30.70 & 53.73 & 23.21 & 0.68 & 32.44 & 76.78 & 46.63 & 39.19 & 72.03 \\
\hline 0.55 & 55.36 & 0.15 & 57.26 & -56.26 & 51.21 & -18.28 & 13.76 & 63.91 & 51.73 & 0.65 & 60.84 & 59.12 & 24.64 & -21.43 & 60.94 & 34.96 & 3.47 & 36.58 & 85.76 & 68.35 & 43.36 & 80.38 \\
\hline 0.60 & 51.97 & 0.12 & 64.06 & -59.14 & 61.50 & -6.33 & 17.26 & 65.59 & 63.97 & 21.40 & 62.78 & 71.68 & 35.04 & -8.65 & 73.40 & 51.95 & 7.10 & 43.16 & 100.86 & 98.37 & 50.18 & 94.59 \\
\hline 0.65 & 48.47 & 0.10 & 73.03 & -61.10 & 76.17 & 9.55 & 21.08 & 70.12 & 80.22 & 48.75 & 67.47 & 88.51 & 49.12 & 12.83 & 93.90 & 79.09 & 11.81 & 53.17 & 125.21 & 144.21 & 60.66 & 117.70 \\
\hline 0.70 & 44.82 & 0.08 & 85.64 & -60.32 & 97.85 & 33.09 & 25.74 & 78.58 & 103.74 & 89.17 & 75.97 & 112.97 & 69.60 & 54.31 & 127.99 & 125.78 & 18.38 & 68.94 & 165.39 & 220.63 & 77.22 & 156.00 \\
\hline 0.75 & 40.96 & 0.06 & 104.09 & -51.03 & 132.04 & 72.90 & 31.61 & 92.59 & 140.38 & 156.24 & 89.93 & 151.17 & 101.62 & 145.36 & 187.94 & 214.32 & 27.95 & 94.76 & 235.30 & 361.19 & 104.37 & 222.95 \\
\hline 0.80 & 36.78 & 0.04 & 132.98 & -14.41 & 191.03 & 150.19 & 39.43 & 115.86 & 202.98 & 282.65 & 113.00 & 216.38 & 156.72 & 377.22 & 304.06 & 405.14 & 42.90 & 140.31 & 368.93 & 654.77 & 152.19 & 351.35 \\
\hline 0.85 & 32.14 & 0.03 & 184.69 & 118.38 & 307.95 & 327.63 & 51.85 & 159.35 & 327.97 & 568.56 & 156.00 & 346.38 & 267.67 & 1101.76 & 567.87 & 900.12 & 70.22 & 233.92 & 670.43 & 1399.17 & 250.19 & 642.10 \\
\hline 0.90 & 26.68 & 0.02 & 295.72 & 751.32 & 604.51 & 883.72 & 73.51 & 252.97 & 640.80 & 1428.25 & 248.54 & 670.69 & 552.06 & 4423.63 & 1367.18 & 2705.01 & 129.35 & 473.55 & 1564.75 & 4016.66 & 500.17 & 1507.48 \\
\hline 95 & 9.51 & 0.01 & 2.26 & 336.43 & 006.12 & 4235.83 & 130.46 & 71.84 & 007.63 & 6432.67 & 563.87 & 081.42 & 1833.02 & 40862.76 & 6038.53 & 16839.24 & 337.27 & 1550.00 & 6681.07 & 23751.36 & 1617.92 & 6477.31 \\
\hline
\end{tabular}


Figures

(a)

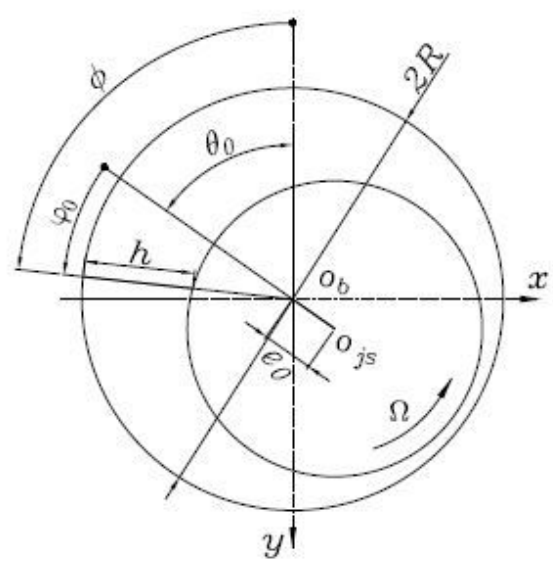

(b)

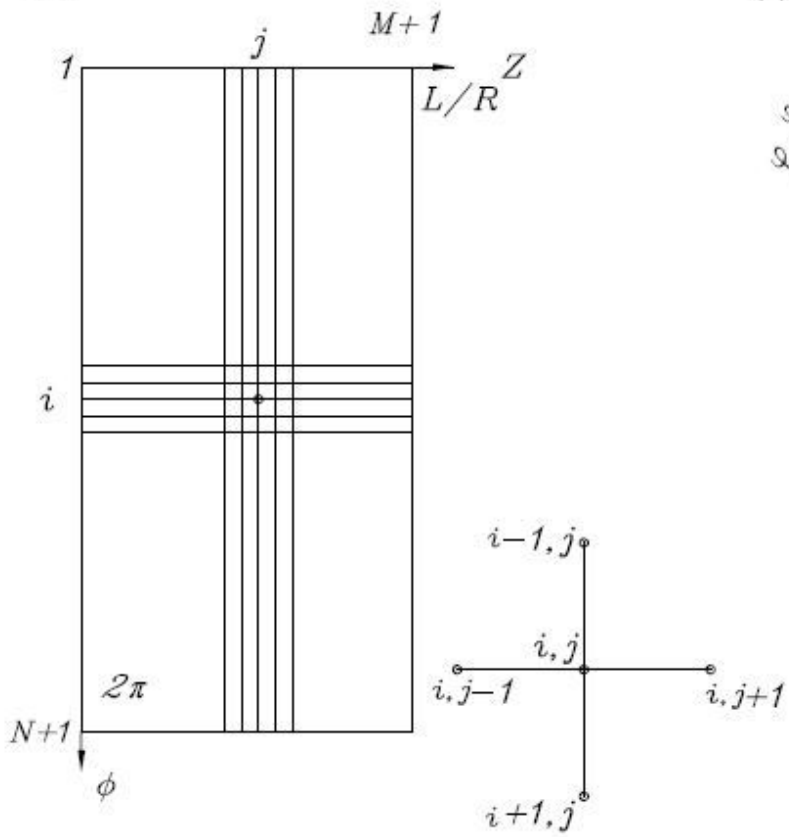

(c)

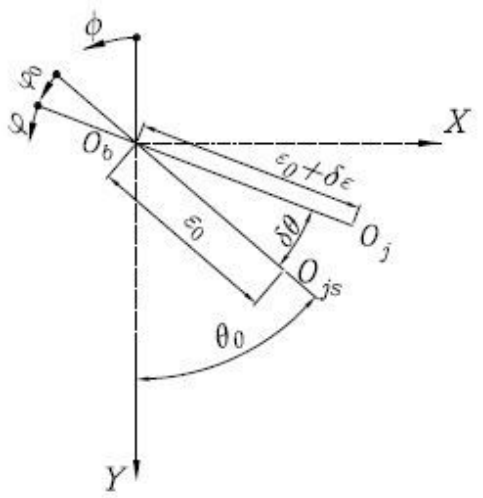

\section{Figure 1}

Full circular journal bearing (a) schematic of the journal bearing coordinates (b) journal bearing mesh (c) perturbed journal bearing in dimensionless coordinates $\mathrm{X}$ and $\mathrm{Y}$

(a)

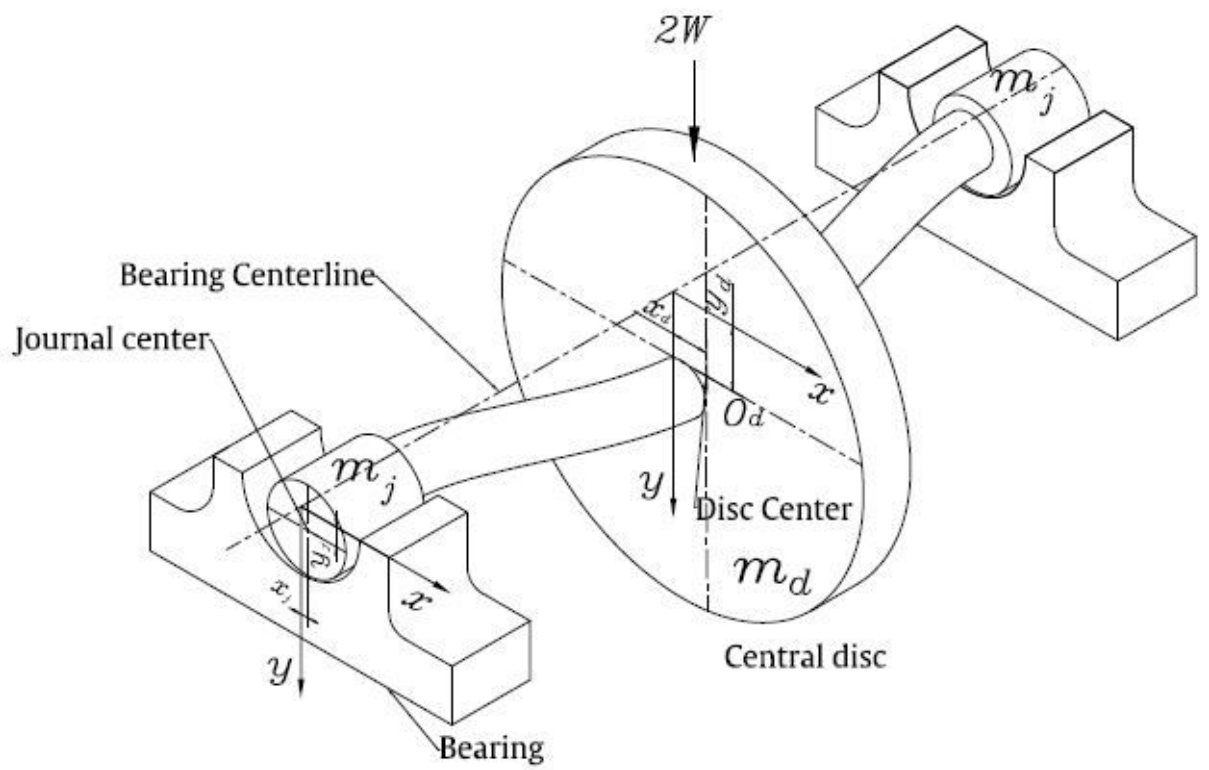

(b)

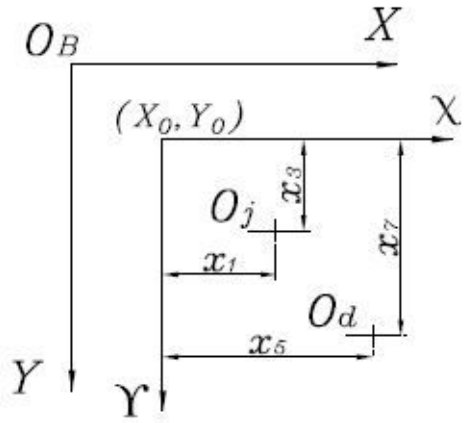

Figure 2 
(a) Elastic rotor supported on two symmetric journal bearings. (b) Bearing coordinates.
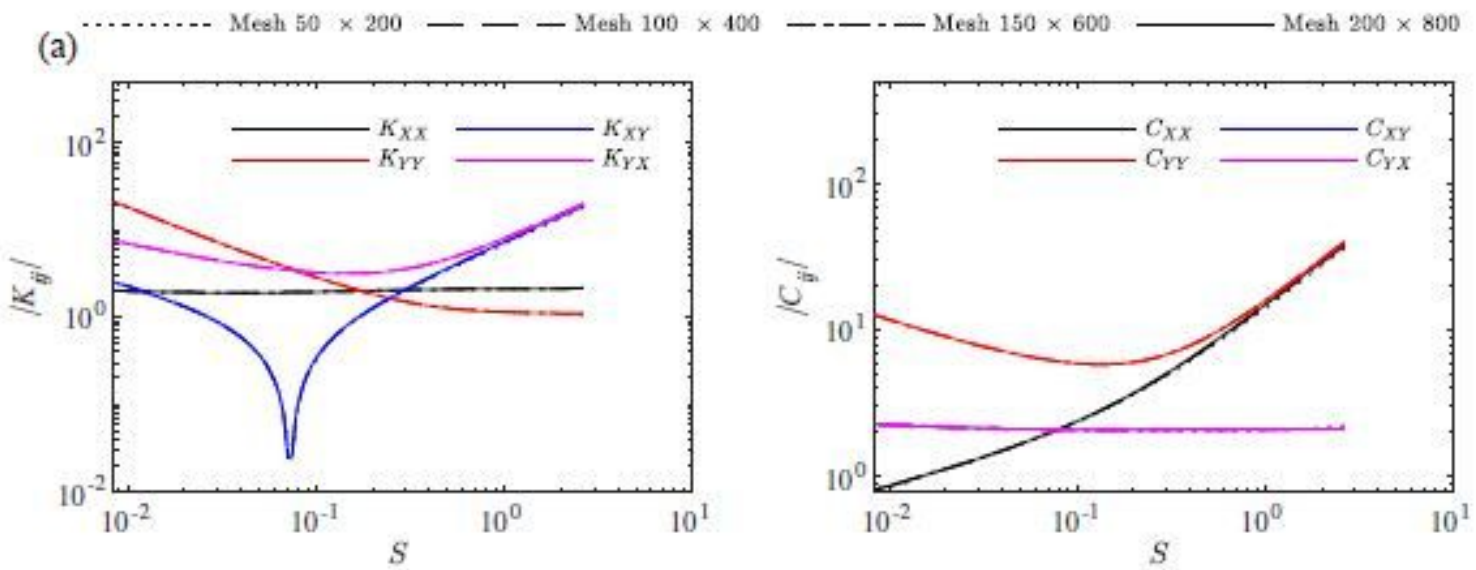

(b)
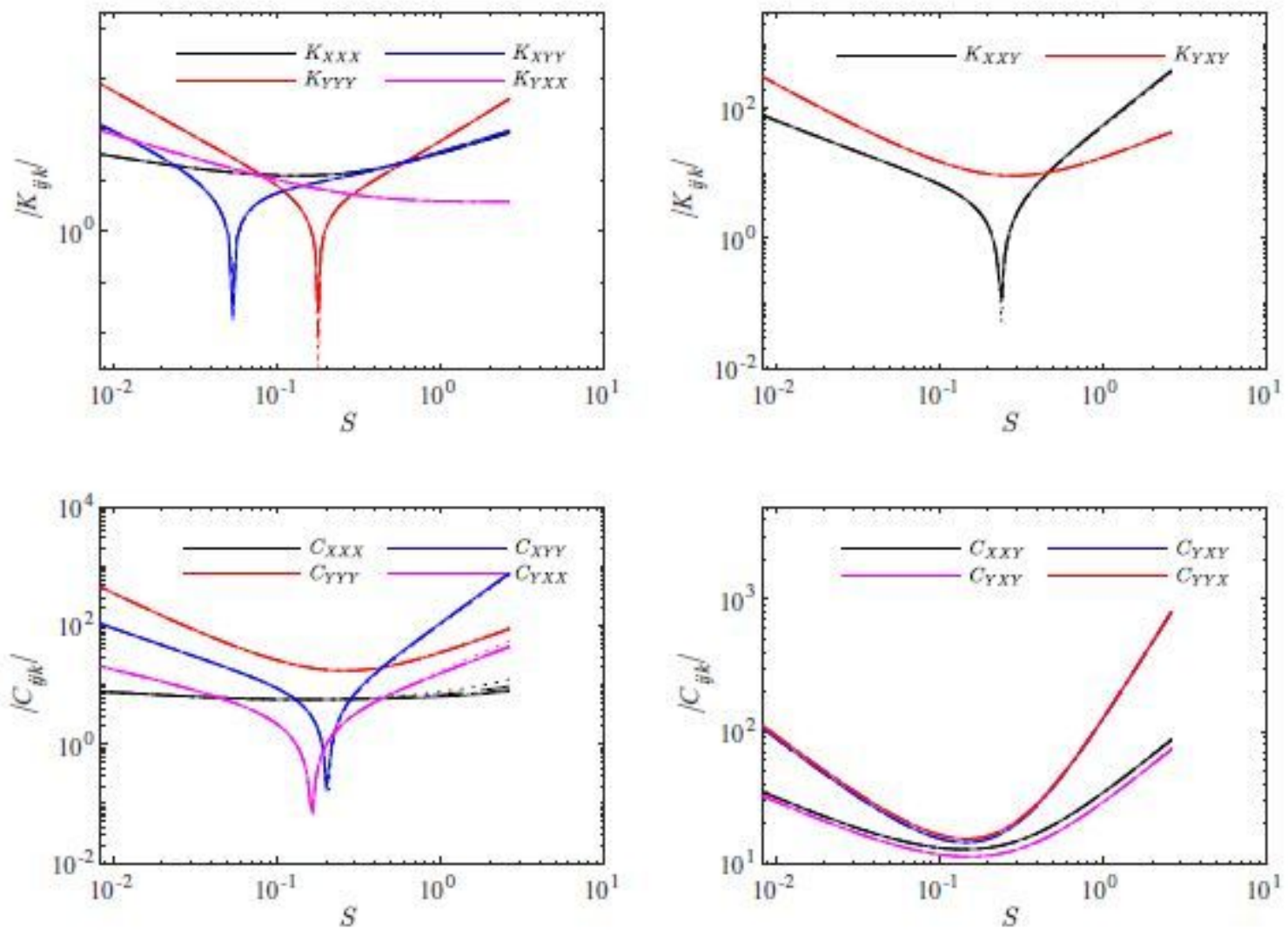

Figure 3

(Color online) Loglog plot for un-grooved journal bearing stiffness and damping coefficients(absolute) versus the Sommerfeld number. $L / D=1$, (a) First order bearing coefficient (b) Second order bearing coefficient. In this gure the dotted line for mesh $50 \times 200$, dashed line for mesh $100 \times 400$, centerline for mesh $150 \times 600$ and solid line for mesh $200 \times 800$. 

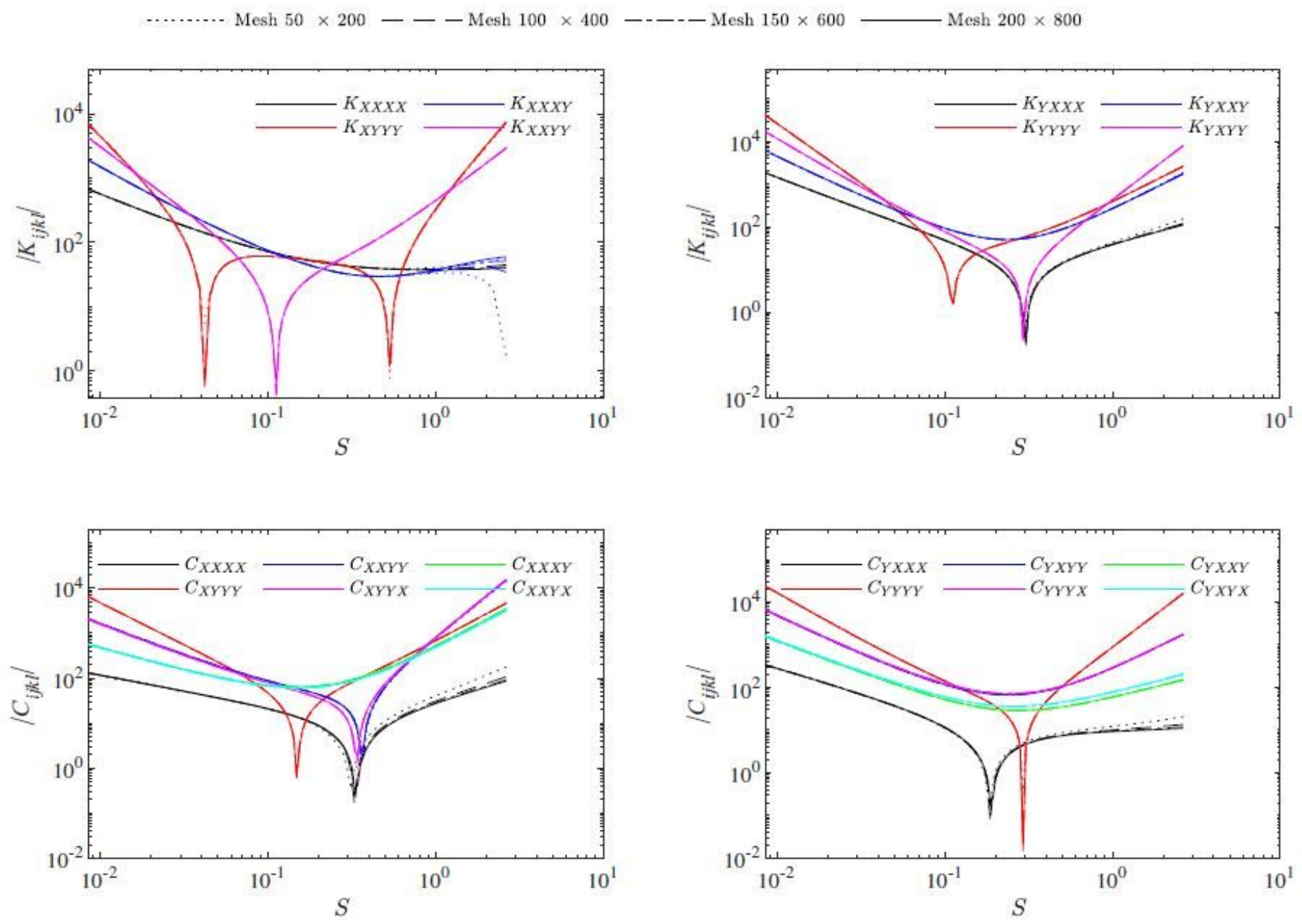

\section{Figure 4}

(Color online) Loglog plot for un-grooved full circular journal bearing third order stiffness and damping coefficients (absolute) versus the Sommerfeld number. L/D ratio is unity. In this gure the dotted line for mesh $50 \times 200$, dashed line for mesh $100 \times 400$, centerline for mesh $150 \times 600$ and solid line for mesh $200 \times 800$ 

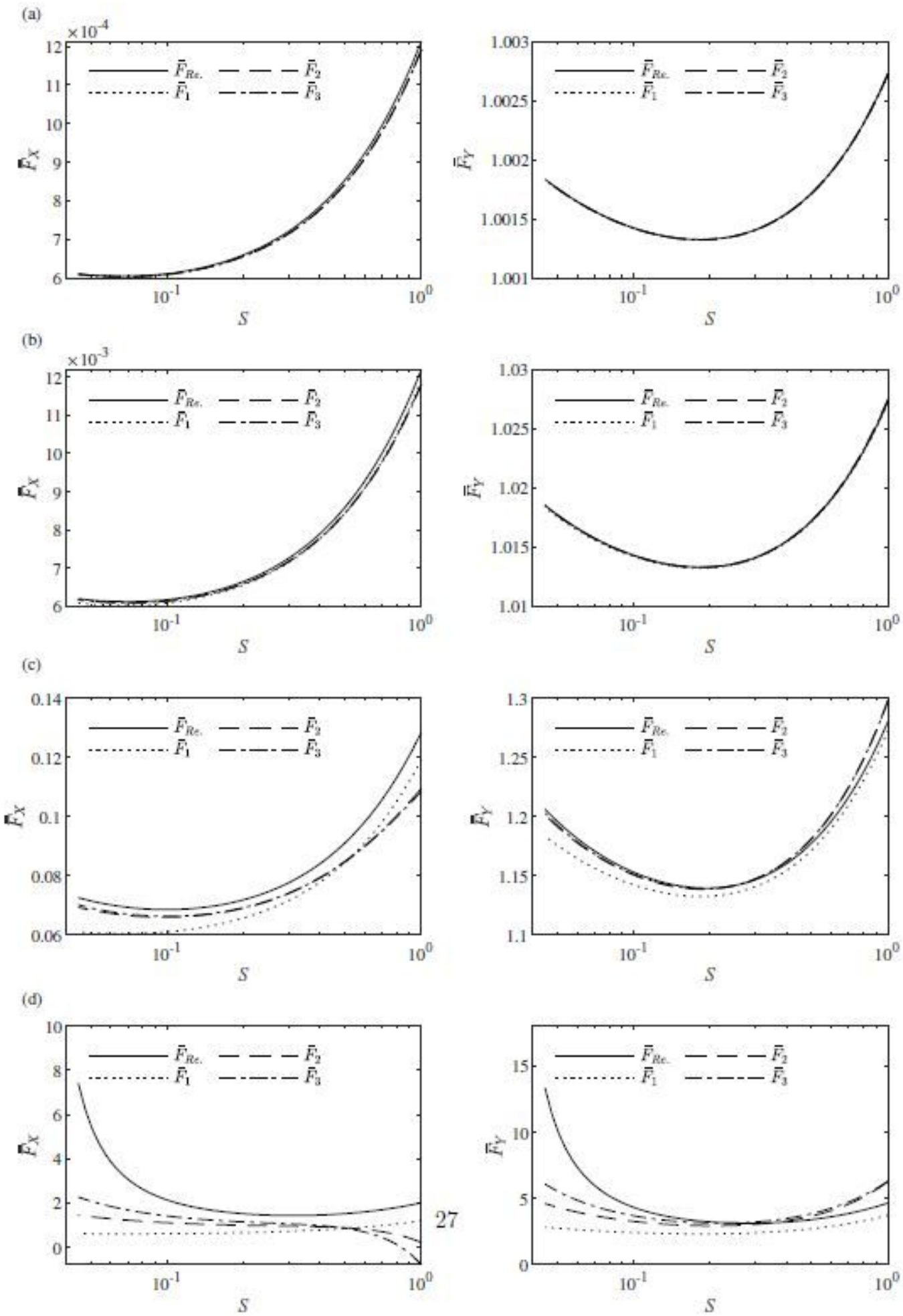

\section{Figure 5}

"Please see the Manuscript PDF file for the complete figure caption". 
(a)

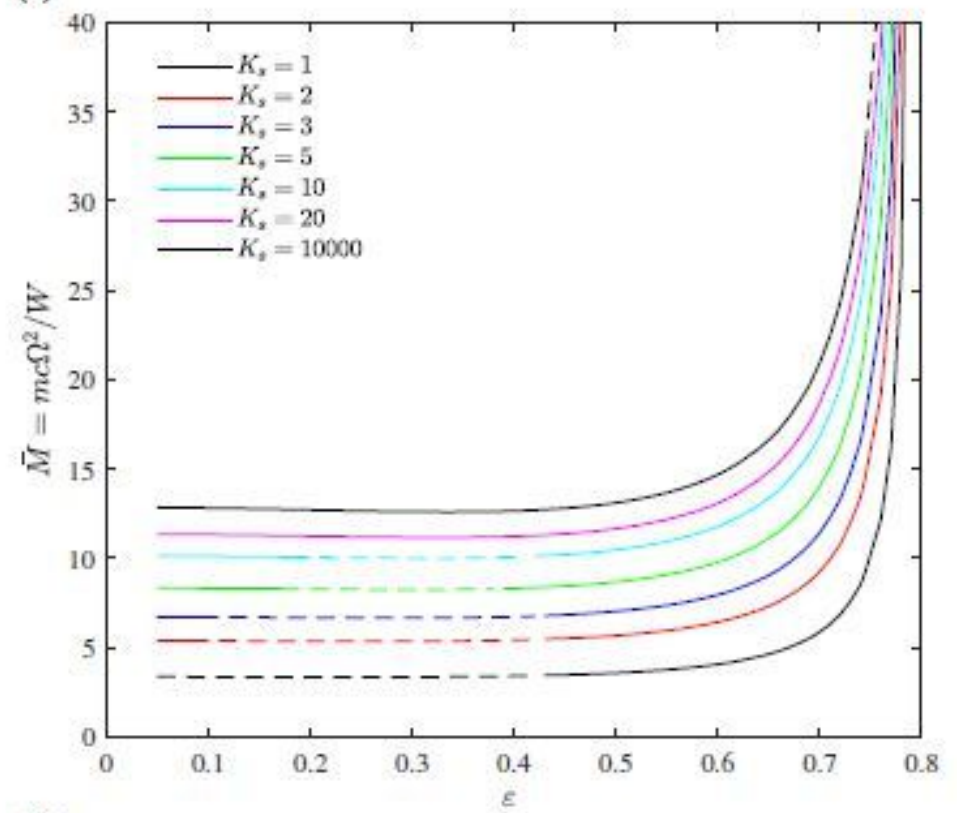

(b)

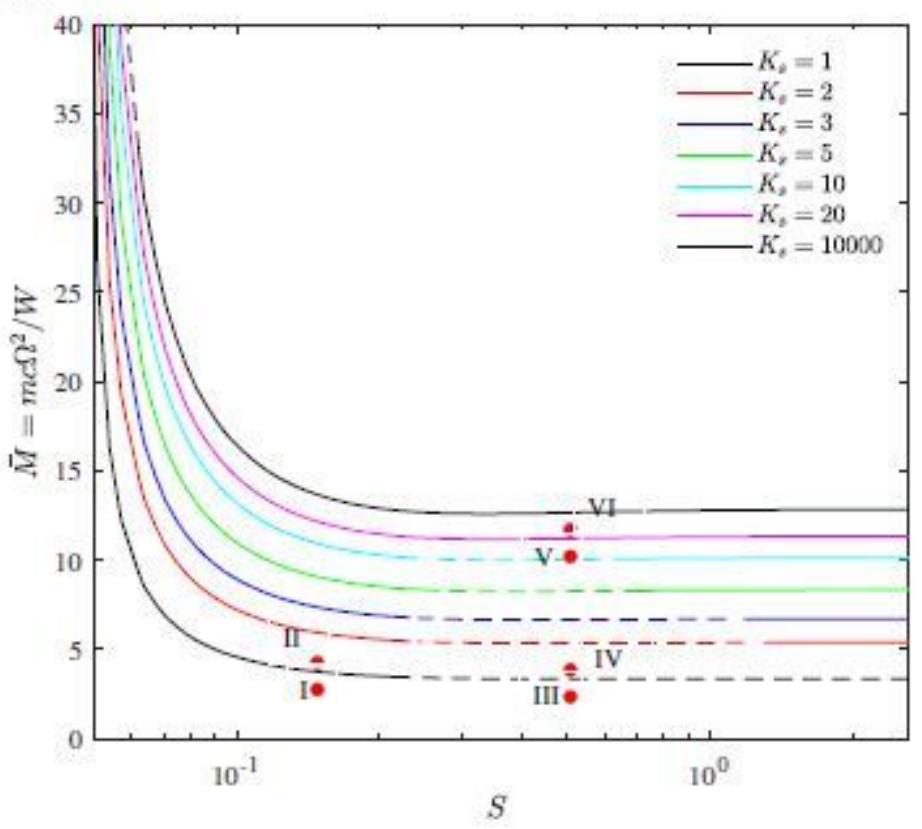

Figure 6

(Color online) Rotor stability curves: (a) Threshold mass versus the eccentricity ratio. (b)Threshold mass versus Sommerfeld No. . 

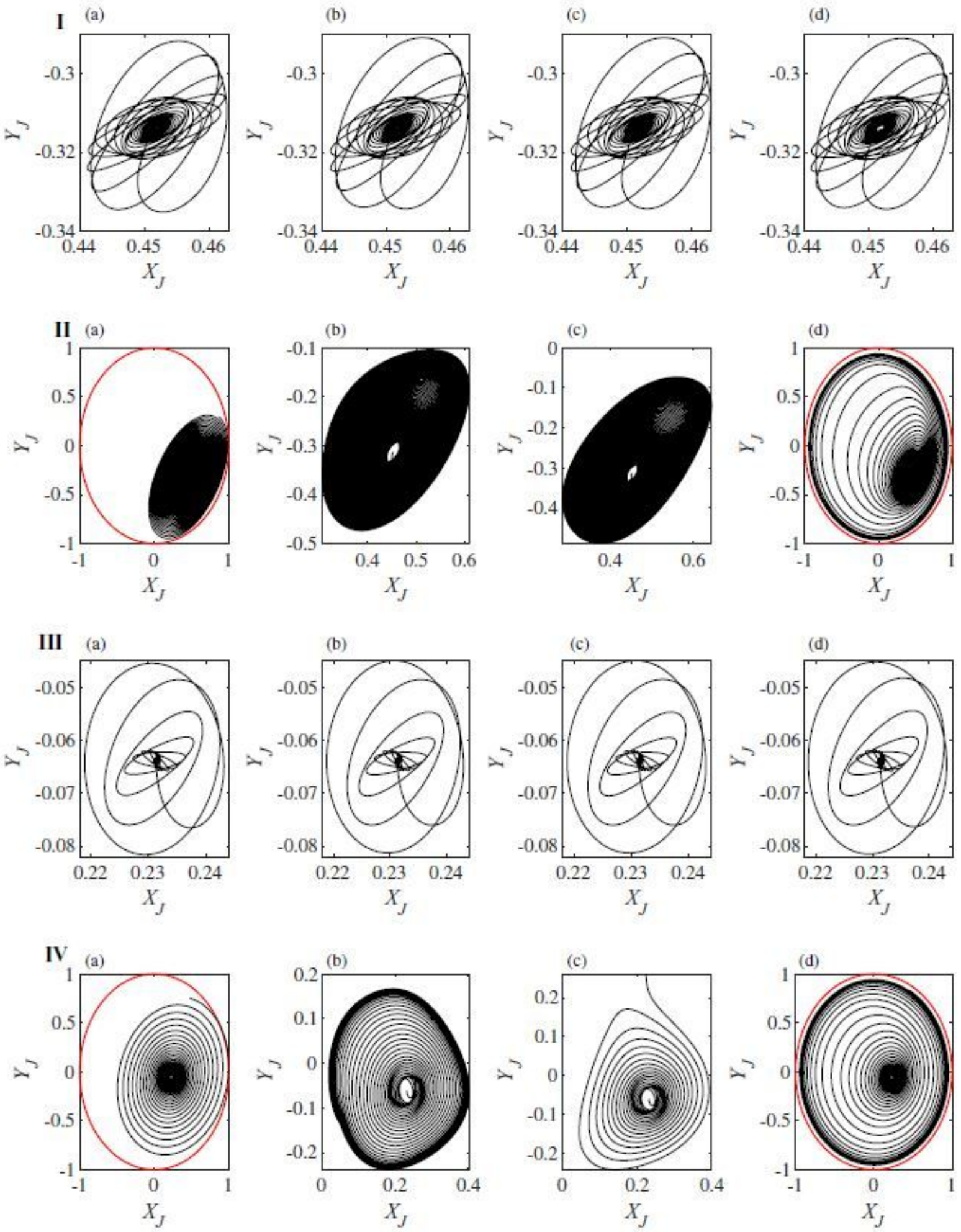

Figure 7

Orbit plot for the journal geometrical center at different operating points, point I, II, III and IV as shown in Table 1. For all cases (a)1st order (LA) (b)2nd order (NLA2) (c) 3rd order(NLA3) (d) nonlinear (RNL). Ks =

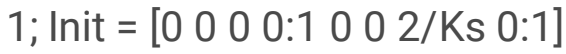




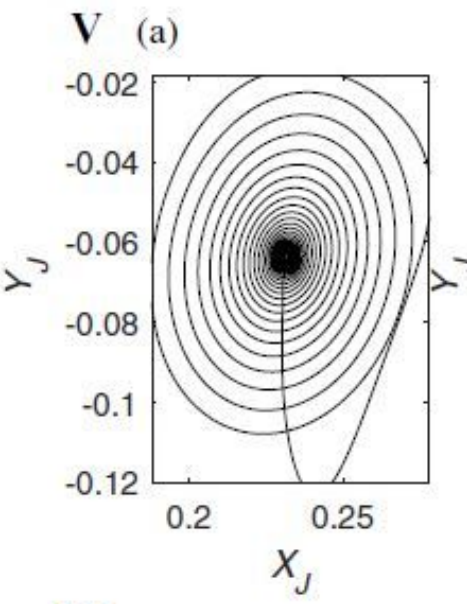

(b)
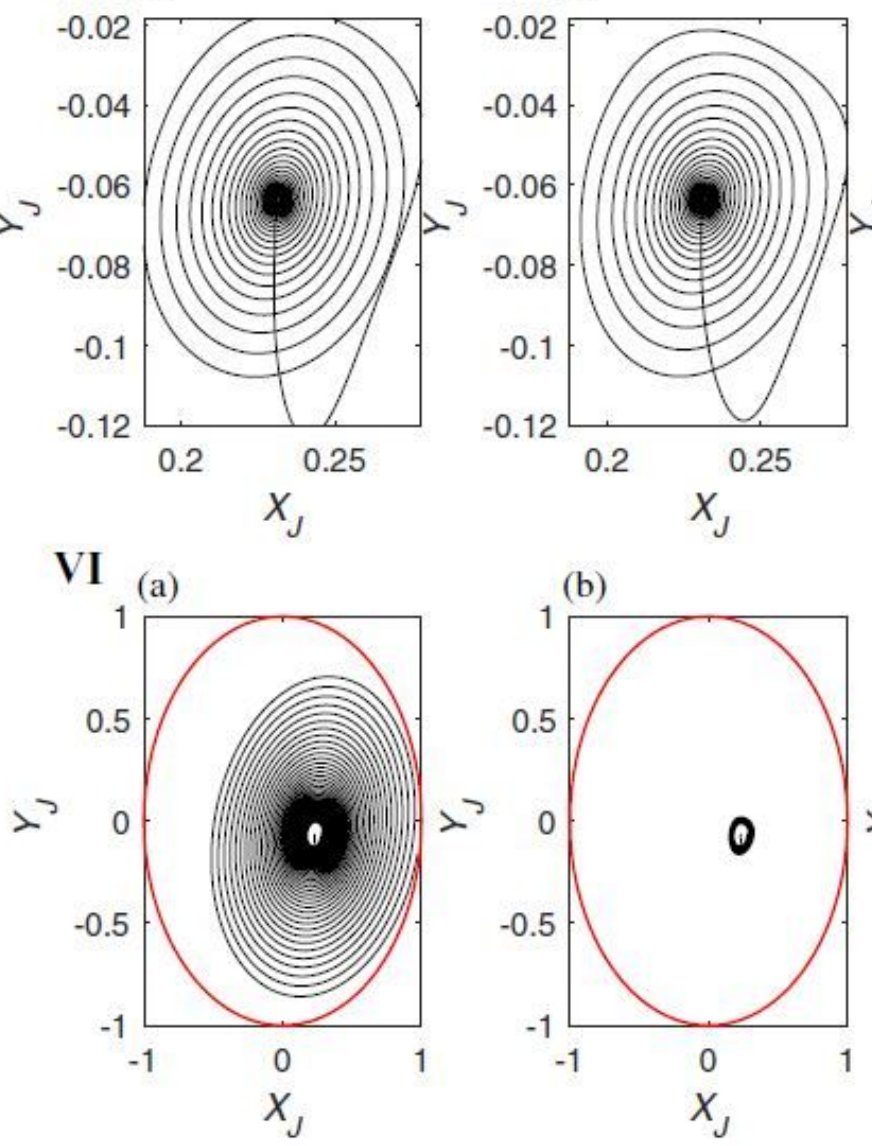

(c)
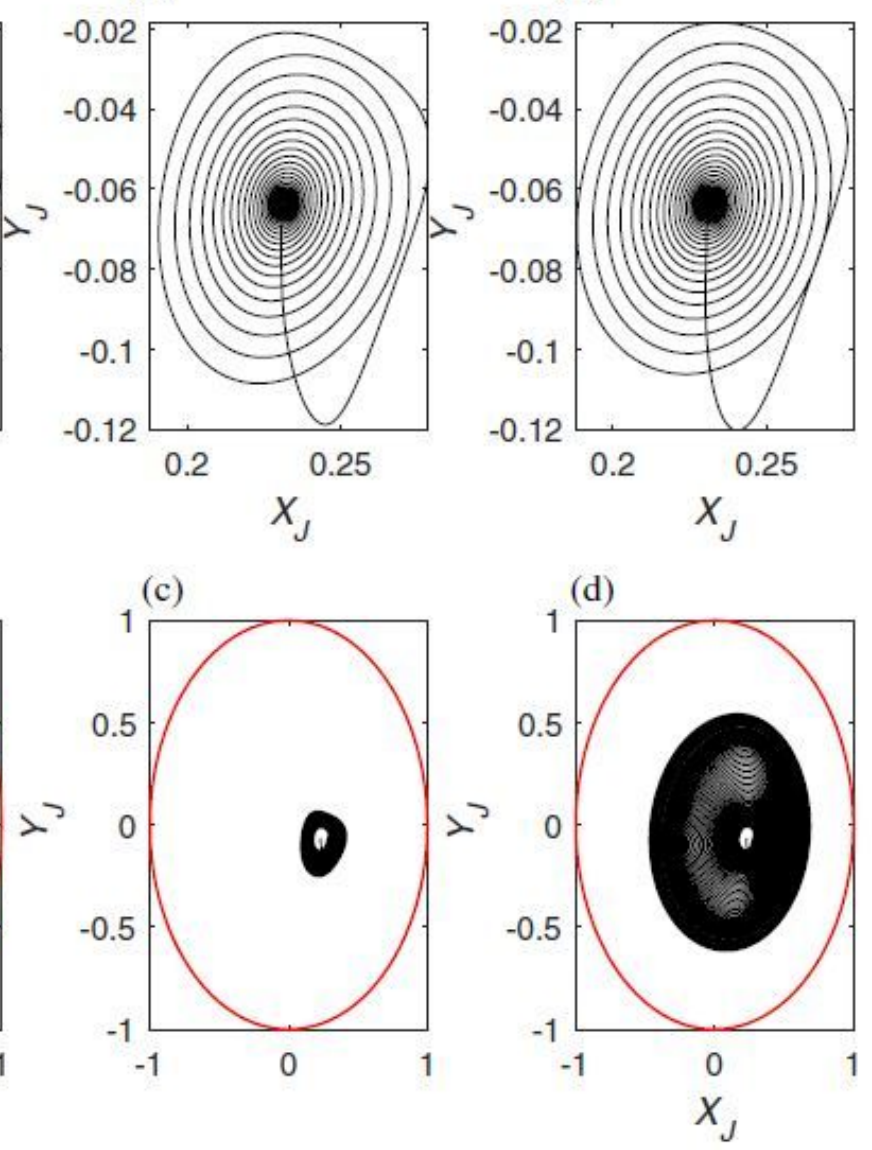

Figure 8

Orbit plot for the journal geometrical center two operating conditions $\mathrm{V}$ and $\mathrm{VI}$ as shown in Table 1. For all cases (a) 1st order (LA) (b) 2nd order (NLA2) (c) 3rd order(NLA3) (d) nonlinear (RNL). Ks = 1; Init = $\left[\begin{array}{lll}0 & 0 & 0\end{array}\right.$ $0: 100$ 2/Ks 0:1] 